Supplement of Geosci. Model Dev., 9, 2271-2278, 2016

http://www.geosci-model-dev.net/9/2271/2016/

doi:10.5194/gmd-9-2271-2016-supplement

(C) Author(s) 2016. CC Attribution 3.0 License.

(c) (i)

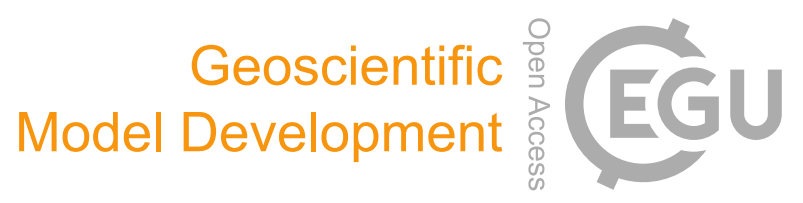

Supplement of

\title{
FABM-PCLake - linking aquatic ecology with hydrodynamics
}

Fenjuan Hu et al.

Correspondence to: Fenjuan Hu (fenjuan.hu@gmail.com)

The copyright of individual parts of the supplement might differ from the CC-BY 3.0 licence. 


\section{Table of Contents}

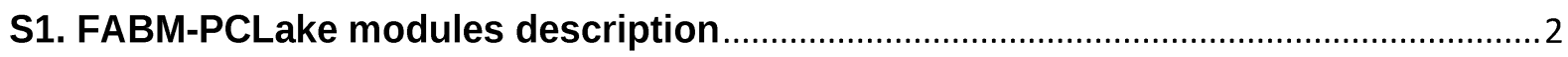

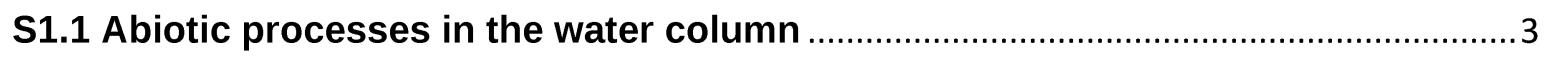

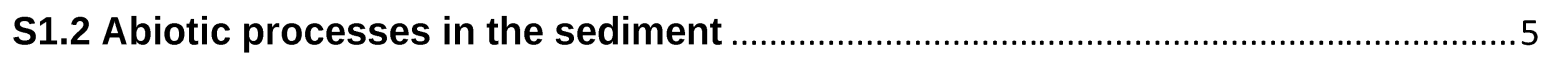

S1.3 Phytoplankton in the water column ............................................................... 7

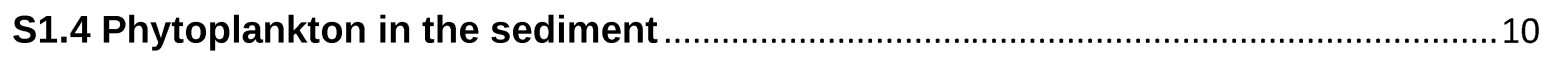

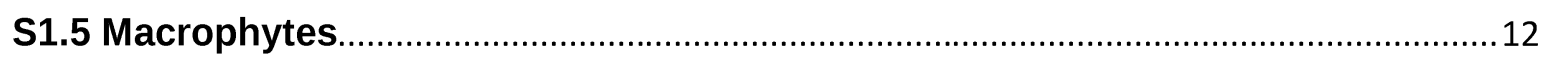

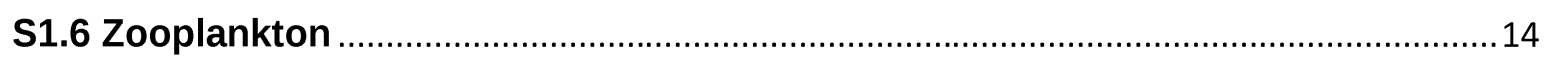

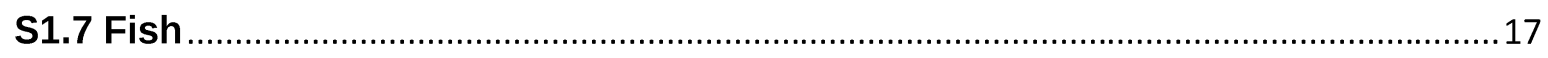

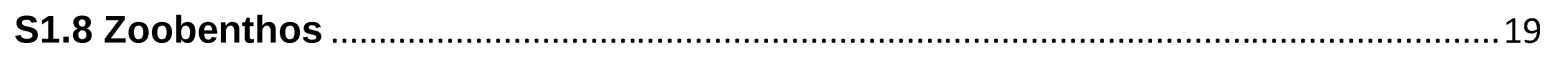

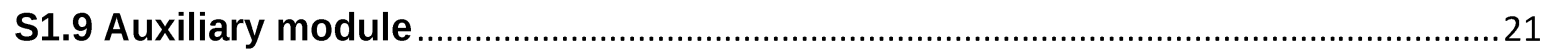

S2. List of FABM-PCLake biogeochemical sink and source terms ..................................24

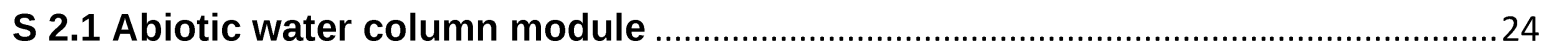

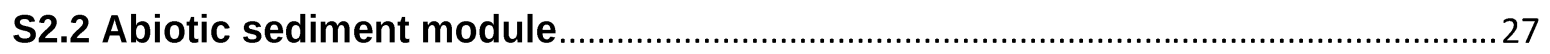

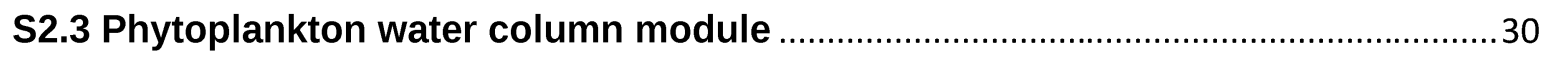

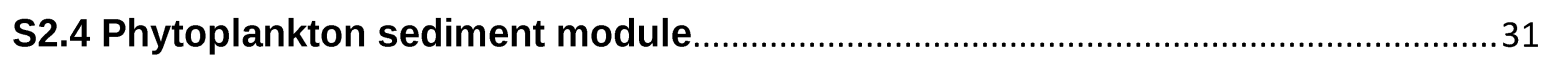

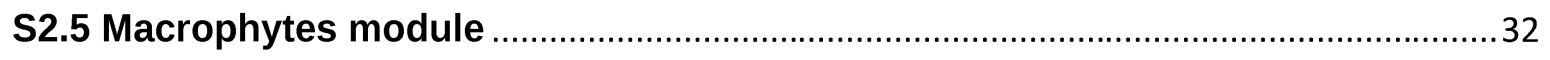

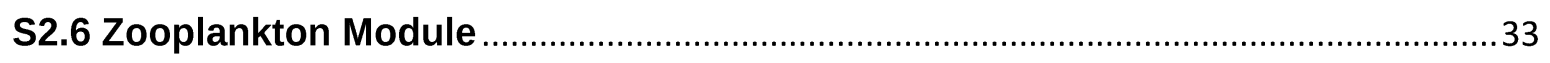

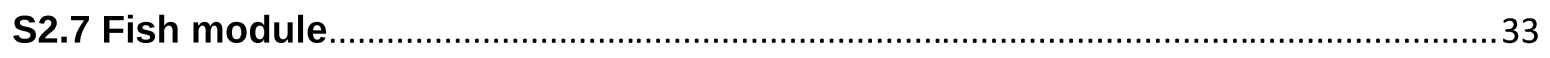

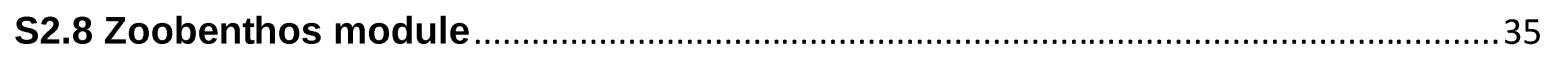

S3. FABM-PCLake parameter list (input file in .yaml format) in modular structure .......36

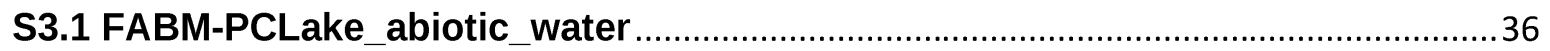

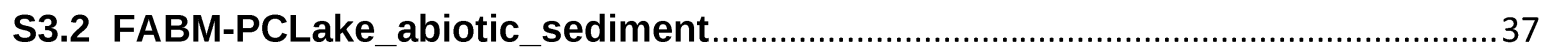

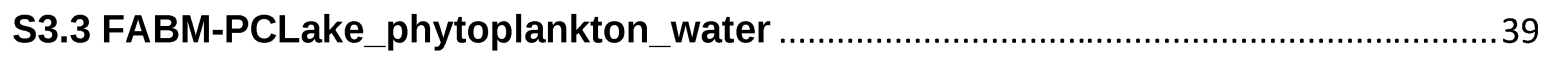

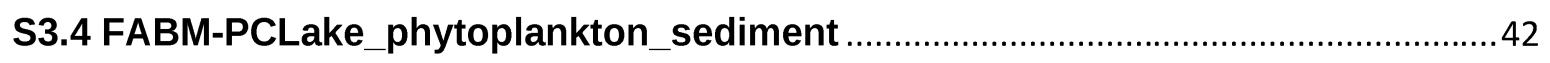

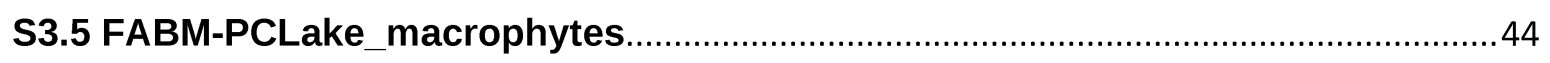

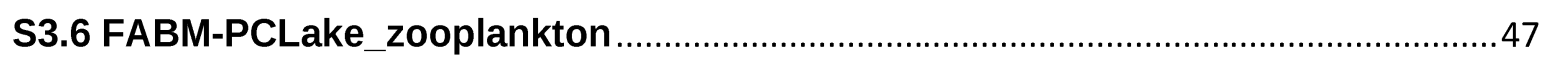

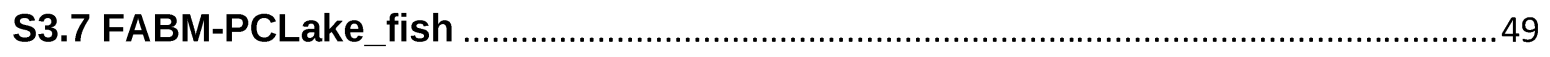

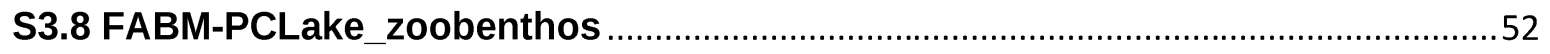

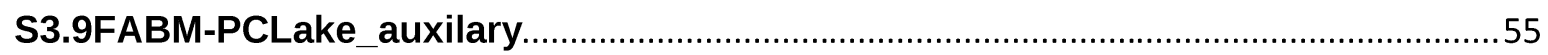

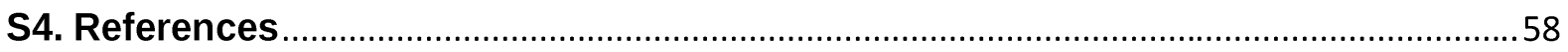




\section{S1. FABM-PCLake modules description}

FABM-PCLake consists of 9 individual modules, and a brief description of each module is provided here.

\section{Supplemental Table S1 Modules overview for FABM-PCLake}

\begin{tabular}{|c|c|c|}
\hline Module & & $\begin{array}{l}\text { Nr. State Processes } \\
\text { variables }\end{array}$ \\
\hline abiotic_water.F90 & 14 & $\begin{array}{l}\text { nitrification, denitrification, mineralisation, } \mathrm{O}_{2} \text { dynamics } \\
\text { in water column }\end{array}$ \\
\hline abiotic_sediment.F90 & 9 & $\begin{array}{l}\text { diffussion, nitrification, denitrification, mineralisation, } \mathrm{O}_{2} \\
\text { demand in sediment }\end{array}$ \\
\hline phytoplankton_water.F90 & 9 & $\begin{array}{l}\text { production, nutrient uptake, respiration, excretion, } \\
\text { mortality }\end{array}$ \\
\hline phytoplankton_sediment.F90 & 9 & respiration, excretion, mortality \\
\hline macrophytes.F90 & 3 & $\begin{array}{l}\text { production, nutrient uptake, respiration, excretion, } \\
\text { mortality, migration, seasonality forcing, germination }\end{array}$ \\
\hline Zooplankton.F90 & 10 & $\begin{array}{l}\text { consumption, assimilation, respiration, excretion, } \\
\text { mortality }\end{array}$ \\
\hline Fish.F90 & & $\begin{array}{l}\text { migration, reproduction, assimilation, excretion, } \\
\text { mortality, consumption, aging }\end{array}$ \\
\hline zoobenthos.F90 & 3 & $\begin{array}{l}\text { consumption, assimilation, respiration, excretion, } \\
\text { mortality }\end{array}$ \\
\hline auxiliary.f90 & 0 & $\begin{array}{l}\text { resuspension, sedimentation, burial, conserved } \\
\text { quantities, mass balance check }\end{array}$ \\
\hline
\end{tabular}


Detailed module descriptions are listed below and adhere to this general structure:

Module description: relevant to corresponding F90 file.

List of variables

- List of local state variables

Local State variables: local state variables are state variables that are registered within this module and changed mainly in this module's processes

- List of diagnostic variables

Diagnostic variables are additional variables that can be output and analysed, and typically these will represent key system properties and provide a more in-depth understanding of the processes occurring in the modules.

- List of external dependencies

The external dependencies are the variables that are provided by other modules, either by the hydrodynamic models (physical drivers) or by other biogeochemical modules (and models) within FABM.

Additional information on the implementation of a biogeochemical model within FABM, e.g., defining state variables, diagnostic variables and external dependencies, is provided on the FABM website (www.fabm.net/wiki) under "Developing_a_new_biogeochemical_model".

\section{S1.1 Abiotic processes in the water column}

\section{File name:}

abiotic_water.F90

\section{Module description:}


The abiotic_water module describes the state variables which are related to abiotic processes in the water column, including: inorganic matter (IM), organic matter (detritus), dissolved nutrients (ammonium, nitrate, phosphate and dissolved silica-dioxide), immobilized phosphorus (adsorbed phosphorus) and dissolved oxygen.

\section{List of variables (in following tables):}

Supplemental Table S2 List of local state variables in abiotic water module

\begin{tabular}{|c|c|}
\hline State variable & Description of state variables \\
\hline sDDIMW & dry weight of inorg. matter in water column, mgDW/l \\
\hline sDDetW & dry weight of detritus in water column, mgDW/l \\
\hline sNDetW & nitrogen in detritus in water column, $\mathrm{mgN} / \mathrm{l}$ \\
\hline sPDetW & phosphorus in detritus in water column,mgP/l \\
\hline sSiDetW & silica in detritus in water column, mgSi/l \\
\hline sPO4W & phosphate in water column,mgP/l \\
\hline SPAIMW & adsorbed phosphorus in water column,mgP/l \\
\hline sNH4W & ammonium in water column, mgN/l \\
\hline sNO3W & nitrate in water column,mgN/l \\
\hline sSiO2W & dissolved silica in water column,mgSi/l \\
\hline $\mathrm{sO} 2 \mathrm{~W}$ & oxygen in water column, $\mathrm{mgO}_{2} / \mathrm{l}$ \\
\hline
\end{tabular}

Supplemental Table S3 List of local diagnostic variables in abiotic water module

\begin{tabular}{ll}
\hline Diagnostic variable & Description of diagnostic variable \\
\hline afO2SatW & afO2SatW $=02$-concentration/saturation O2- \\
& concentration $* 100$, oxygen saturation percentage,$\%$ \\
rNDDetW & $r N D D e t W=s N D e t W / s D D e t W, N / D$ ratio of detritus in water,$g N / g D$ \\
rPDDetW & $r P D D e t W=s P D e t W / s D$ Det $W, P / D$ ratio of detritus in water, \\
& $g P / g D$
\end{tabular}




\begin{tabular}{ll}
\hline Diagnostic variable & Description of diagnostic variable \\
\hline extIM & extIM $=s D I M W^{*} c E x t S p I M$, extinction factor caused by inorganic \\
& matter, - \\
extDet & extDet=sDDetW*CEtSpDet, extinction factor caused by organic \\
& matter,- \\
tO2Aer & oxygen reaeration rate, mg/l/day \\
\hline
\end{tabular}

Supplemental Table S4 List of dependencies in abiotic water column module

\begin{tabular}{llll}
\hline dependencies & Description & Linked external module & Linked external \\
& & & variable \\
\hline uTm & temperature & physical driver & temperature \\
uVWind & wind speed & physical driver & wind_speed \\
\hline
\end{tabular}

S1.2 Abiotic processes in the sediment

File name: abiotic_sediment.F90

Module description: The abiotic_sediment module describes all the state variables which are related to abiotic processes in the sediment, including: inorganic matter (IM), organic matter (detritus), dissolved nutrients (ammonium, nitrate, and phosphate), immobilized phosphorus (adsorbed phosphorus) and humus.

\section{List of variables (in following tables):}

Supplemental Table S5 List of local state variables in abiotic sediment module

\begin{tabular}{ll}
\hline State variable & Description of state variables \\
\hline sDDIMS & dry weight of inorg. matter in sediment, gDW/m**2 \\
sDDetS & dry weight of detritus in sediment, gDW $/ \mathrm{m}^{\star * 2}$ \\
sNDetS & nitrogen in detritus in sediment, $\mathrm{gN} / \mathrm{m}^{\star \star 2}$ \\
sPDetS & phosphorus in detritus in sediment, $g P / \mathrm{m}^{\star \star 2}$
\end{tabular}




\begin{tabular}{|c|c|}
\hline State variable & Description of state variables \\
\hline sSiDetS & silica in detritus in sediment, $g \mathrm{Si} / \mathrm{m}^{\star \star 2}$ \\
\hline sPO4S & phosphate in sediment,gP/m*2 \\
\hline SPAIMS & adsorbed phosphorus in sediment,gP/**2 \\
\hline sNH4S & ammonium in sediment, $\mathrm{gN} / \star \star 2$ \\
\hline sNO3S & nitrate in sediment, $g N / m^{\star * 2}$ \\
\hline sDHums & dry weight of humus in sediment, gDW/m**2 \\
\hline sNHumS & nitrogen of humus in sediment, $g N / m^{\star \star 2}$ \\
\hline sPHumS & phosphorus of humus in sediment, gP/m² \\
\hline
\end{tabular}

Supplemental Table S6 List of local diagnostic variables in abiotic water module

\begin{tabular}{ll}
\hline diagnostic variable & Description of diagnostic variable \\
\hline afOxySed & afOxySed = aDepthOxySed / cDepthS, proportion aerobic \\
& sediment, - \\
rNDDetS & rNDDetS = sNDetS / sDDetS, N content of sediment detritus, \\
& gN/gDW \\
rPDDetS & rPDDetS = sPDetS / sDDetS, P content of sediment detritus, \\
& gP/gDW \\
& $a P E q I M S=a P / s o A d s S * s D I M S$, equilibrium amount in sediment, \\
aPEqIMS & $\mathrm{gP} / \mathrm{m}^{\star * 2}$
\end{tabular}

Supplemental Table S7 List of dependencies in abiotic sediment module

dependencies Description Linked external Linked external




\begin{tabular}{llll}
\hline & & module & variable \\
\hline uTm & temperature & physical driver & temperature \\
SWNH4 & ammonium_pool_in_water & abiotic_water & sNH4W \\
SWNO3 & nitrate_pool_in_water & abiotic_water & sNO3W \\
SWPO4 & phosphate_pool_in_water & abiotic_water & sPO4W \\
SWSiO2 & siO2_release_to_water & abiotic_water & sSiO2W \\
O2Supply & oxygen_pool & abiotic_water & sO2W \\
\hline
\end{tabular}

\section{S1.3 Phytoplankton in the water column}

File name: phytoplankton_water.F90

\section{Module description:}

The phytoplankton_water module describes the processes related to phytoplankton in the water column.Three groups of phytoplankton are described here: diatoms, green algae and cyanobacteria (blue-green algae). Each group is described in three elements, dry weight, nitrogen and phosphorus. The silica concentration in diatoms is not a state variables here but instead defined as a diagnostic variable, since the model assumes that diatoms have a fixed Si/DW ratio, i.e., 0.1.

\section{List of variables (in following tables):}

Supplemental Table S8 List of local state variables in phytoplankton water module

\begin{tabular}{ll}
\hline State variable & Description of state variables \\
\hline sDDiatW & diatom concentration in dry weight, mgDW/l \\
sPDiatW & diatom concentration in phosphorus, mgP/l \\
sNDiatW & diatom concentration in nitrogen, mgN/l \\
sDGrenW & green algae concentration in dry weight, mgDW/l \\
sPGrenW & green algae concentration in phosphorus, mgP/l
\end{tabular}




\begin{tabular}{ll}
\hline sNGrenW & green algae concentration in nitrogen, mgN/l \\
sDBlueW & blue-green algae concentration in dry weight, mgDW/l \\
sPBlueW & blue-green algae concentration in phosphorus, mgP/l \\
sNBlueW & blue-green algae concentration in nitrogen, $\mathrm{mgN} / \mathrm{l}$ \\
\hline
\end{tabular}

\section{Supplemental Table S9 List of local diagnostic variables in phytoplankton water module}

\begin{tabular}{|c|c|}
\hline diagnostic variable & Description of diagnostic variable \\
\hline oChlaBlue & blue-green algae chlorophyll-a concentration,ug/l \\
\hline aLLimBlue & light limitation factor for blue-green algae,-- \\
\hline aNutLimBlue & nutrient limitation factor for blue-green algae \\
\hline extBlue & $\begin{array}{l}\text { extBlue }=s D B / u e W^{\star} c E x t S p / M, \text { light extinction factor caused by } \\
\text { blue-green algae, } \mathrm{m}-1\end{array}$ \\
\hline rNDBlueW & $\begin{array}{l}r N D B l u e W=s N B l u e W / s D B l u e W, N \text { content in blue-green } \\
\text { algae,gN/gD }\end{array}$ \\
\hline rPDBlueW & $\begin{array}{l}r P D B / u e W=s P B / u e W / s D B l u e W, P \text { content in blue-green } \\
\text { algae,gP/gD }\end{array}$ \\
\hline oChlaGren & green algae chlorophyll-a concentration,ug/l \\
\hline aLLimGren & light limitation factor for green algae,- \\
\hline aNutLimGren & nutrient limitation factor for green algae,-- \\
\hline extGren & $\begin{array}{l}\text { extGren }=s D G r e n W^{*} c E x t S p I M, \text { light extinction factor caused by } \\
\text { green algae,m-1 }\end{array}$ \\
\hline rNDGrenW & $r N D G r e e n W=s N G r e n W / s D G r e n W, N$ content in green algae, $g N / g D$ \\
\hline rPDGrenW & $r P D G r e e n W=s P G r e n W / s D G r e n W, P$ content in green algae, $\mathrm{gP} / \mathrm{gD}$ \\
\hline oChlaDiat & diatom chlorophyll-a concentration,ug/l \\
\hline aLLimDiat & light limitation factor for diatoms,-- \\
\hline aNutLimDiat & nutrient limitation factor for diatoms,-- \\
\hline
\end{tabular}




\begin{tabular}{|c|c|}
\hline diagnostic variable & Description of diagnostic variable \\
\hline extDiat & $\begin{array}{l}\text { extDiat }=s D \text { Diat } W^{*} c E x t S p / M \text {,light extinction factor caused by } \\
\text { diatoms, } \mathrm{m}^{-1}\end{array}$ \\
\hline rNDDiatW & $r N D D i a t W=s N D i a t W / s D D i a t W, N$ content in diatoms, gN/gDW \\
\hline rPDDiatW & $r P D D i a t W=s P D i a t W / s D D i a t W, \mathrm{P}$ content in diatoms, $\mathrm{gP} / \mathrm{gDW}$ \\
\hline oSiDiatW & oSiDiatW=sDDiat $W^{*} c S i D D i a t W$, diatom silica concentration, mgSi/l \\
\hline aDPhytw & $\begin{array}{l}\text { aDPhytW=sDBlueW+sDGrenW+sDDiatW,total algae biomass in } \\
\text { the water column,mgDW/l }\end{array}$ \\
\hline rNDPhytW & $\begin{array}{l}r N D P h y t W=(s N B l u e W+s N G r e n W+s N D i a t W) / a D P h y t W, N \text { content } \\
\text { in phytoplankton in water column, gN/gDW }\end{array}$ \\
\hline rPDPhytw & $\begin{array}{l}r P D P h y t W=(s P B / \text { ueW }+s P G r e n W+s P D i a t W) / a D P h y t W, P \text { content in } \\
\text { phytoplankton in water column, gP/gDW }\end{array}$ \\
\hline ParSurf & surface photosynthetically active radiation, $\mathrm{W} / \mathrm{m}^{\star \star 2}$ \\
\hline phypar & local photosynthetically active radiation (layer center par), W/m*2 \\
\hline phytoextinction & local extinction factor used by phytoplankton light function, $\mathrm{m}^{-1}$ \\
\hline wO2PrimW & oxygen production by phytoplankton, mgO2///d \\
\hline
\end{tabular}

Supplemental Table S10 List of dependencies in phytoplankton water module

\begin{tabular}{llll}
\hline dependencies & Description & Linked external & Linked external variable \\
& & module & \\
\hline uTm & Temperature & physical driver & temperature \\
par & photosynthetically & physical driver & downwelling_photosynthetic \\
& active radiation & & _radiative_flux \\
Dz & cell_thickness & physical driver & cell_thickness \\
extc & attenuation coefficient & physical driver & attenuation_coefficient_of_p \\
& & & hotosynthetic_radiative_flux
\end{tabular}




\begin{tabular}{llll}
\hline dependencies & Description & Linked external & Linked external variable \\
& & module & \\
\hline SiO2poolW & $\mathrm{SiO}_{2}$ source_wat & abiotic_water & sSiO2W \\
PO4poolW & sPO4_source_wat & abiotic_water & sPO4W \\
NO3poolW & sNO3_source_wat & abiotic_water & sNO3S \\
NH4poolW & sNH4_source_wat & abiotic_water & sNH4W \\
O2poolW & oxygen_pool & abiotic_water & sO2W \\
DDetpoolW & detritus_DW_pool_wat & abiotic_water & sDDetW \\
NDetpoolW & detritus_N_pool_wat & abiotic_water & sNDetW \\
PDetpoolW & detritus_P_pool_wat & abiotic_water & sPDetW \\
SiDetpoolW & detritus_Si_pool_wat & abiotic_water & sSiDetW \\
\hline
\end{tabular}

\section{S1.4 Phytoplankton in the sediment}

\section{File name:}

phytoplankton_sediment.F90

\section{Module description:}

The phytoplankton_sediment module describes the processes related to the phytoplankton which has settled to the bottom sediments. Three groups of phytoplankton are described here: diatoms, green algae and cyanobacteria (blue-green algae). Each group is described in three elements, dry weight, nitrogen and phosphorus. The silica concentration in diatom is not a state variable here but instead a diagnostic variable, since the model assumes that diatoms have a fixed Si/DW ratio, i.e., 0.1

\section{List of variables (in following tables):}

Supplemental Table S11 List of local state variables in phytoplankton sediment module 


\begin{tabular}{|c|c|}
\hline State variable & Description of state variables \\
\hline sDDiatS & diatom concentration in dry weight,gDW/m**2 \\
\hline sPDiatS & diatom concentration in phosphorus,gP/m**2 \\
\hline sNDiatS & diatom concentration in nitrogen, $g N / m^{\star \star 2}$ \\
\hline sDGrens & green algae concentration in dry weight,gDW/m**2 \\
\hline sPGrenS & green algae concentration in phosphorus, $g \mathrm{P} / \mathrm{m}^{\star \star 2}$ \\
\hline sNGrens & green algae concentration in nitrogen, $g N / m^{\star \star 2}$ \\
\hline sDBlues & blue-green algae concentration in dry weight,gDW/m**2 \\
\hline sPBlues & blue-green algae concentration in phosphorus,gP/m*2 \\
\hline sNBlues & blue-green algae concentration in nitrogen,gN/m**2 \\
\hline
\end{tabular}

Supplemental Table S12 List of local diagnostic variables in phytoplankton sediment module

\begin{tabular}{ll}
\hline Diagnostic variable & Description of diagnostic variable \\
\hline oSiDiatS & sediment diatom concentration in silica, gSi/m² \\
rNDPhytS & $r N D P h y t S=($ sNBlueS + sNGrenS+sNDiatS)/aDPhytS, N content in \\
& phytoplankton in sediment,gN/gD \\
& $r P D P h y t S=(s P B l u e S+s P G r e n S+s P D i a t S) / a D P h y t S, P$ content in \\
rPDPhytS & phytoplankton in sediment,gP/gD \\
\hline
\end{tabular}

Supplemental Table S13 List of dependencies in the phytoplankton sediment module dependencie Description Linked external Linked external 


\begin{tabular}{llll}
\hline s & & module & variable \\
\hline uTm & temperature & physical driver & temperature \\
PO4poolS & sPO4_source_sed & abiotic_sediment & sPO4S \\
NO3poolS & sNO3_source_sed & abiotic_sediment & sNO3S \\
NH4poolS & sNH4_source_sed & abiotic_sediment & sNH4S \\
DDetpoolS & detritus_DW_pool_sed & abiotic_sediment & sDDetS \\
NDetpoolS & detritus_N_pool_sed & abiotic_sediment & sNDetS \\
PDetpoolS & detritus_P_pool_sed & abiotic_sediment & sPDetS \\
SiDetpoolS & detritus_Si_pool_sed & abiotic_sediment & sSiDetS \\
SiO2poolW & SiO 2 source_sed & abiotic_water & sSiO2W \\
\hline
\end{tabular}

\section{S1.5 Macrophytes}

\section{File name:}

macrophytes.F90

\section{Module description:}

This module describes the submerged macrophytes group, and is implemented as a benthic module. The state variables include: sDVeg, sNVeg, sPVeg (vegetation mass in dry weight, nitrogen and phosphorus, respectively). The related processes are: assimilation (only for sDVeg), nutrient uptake (only for sNVeg and sPVeg), respiration (only for sDVeg), excretion (only for sNVeg and sPVeg), mortality and migration.

\section{List of variables (in following tables):}

Supplemental Table S14 List of local state variables in macrophytes module

\section{State variable Description of state variables}

sDVeg vegetation (shoots+roots) in dry weight,gDW/m² 
sNVeg vegetation (shoots+roots) in nitrogen, $g N / m^{\star \star 2}$

sPVeg vegetation (shoots+roots) in phosphorus, $g \mathrm{P} / \mathrm{m}^{\star \star 2}$

Supplemental Table S15 List of local diagnostic variables in macrophytes module

\begin{tabular}{ll}
\hline $\begin{array}{l}\text { diagnostic } \\
\text { variable }\end{array}$ & Description of diagnostic variable \\
\hline aCovVeg & coverage of macrophytes, \% \\
aDSubVeg & submerged macrophytes in dry weight,gDW/m**2 \\
aLPAR1Veg & photosynthetically active radiation at top of vegetation layer, W/m**2 \\
aLPAR2Veg & photosynthetically active radiation at bottom of vegetation layer, \\
& W/m**2 \\
aNutLimVeg & nutrient limitation function for macrophytes,- \\
aLLimVeg & light limitation function for macrophytes,- \\
macroextinction & local extinction factor used by macrophytes light function, $\mathrm{m}-1$
\end{tabular}

Supplemental Table S16 List of dependencies in macrophytes module

\begin{tabular}{llll}
\hline dependencie & Description & Linked external & Linked external \\
s & & module & variable \\
parm & temperature & physical driver & temperature \\
& photosynthetically active & physical driver & downwelling_phot \\
& radiation & & osynthetic_radiati \\
dz & & & ve_flux \\
sDepthW & water depth & physical driver & cell_thickness \\
extc & attenuation coefficient & physical driver & bottom_depth \\
& & & attenuation_coeffi \\
& & & cient_of_photosyn
\end{tabular}




\begin{tabular}{|c|c|c|c|}
\hline $\begin{array}{l}\text { dependencie } \\
\text { s }\end{array}$ & Description & $\begin{array}{l}\text { Linked external } \\
\text { module }\end{array}$ & $\begin{array}{l}\text { Linked external } \\
\text { variable }\end{array}$ \\
\hline day & $\begin{array}{l}\text { number of days since start of } \\
\text { the year }\end{array}$ & physical driver & $\begin{array}{l}\text { ux } \\
\text { number_of_days_ } \\
\text { since_start_of_the } \\
\text { _year }\end{array}$ \\
\hline NH4poolW & $\begin{array}{l}\text { uptake_target_Ammonium_ } \\
\text { water }\end{array}$ & abiotic_water & $\mathrm{sNH} 4 \mathrm{~W}$ \\
\hline NO3poolW & uptake_target_Nitrate_water & abiotic_water & sNO3W \\
\hline PO4poolW & $\begin{array}{l}\text { uptake_target_Phosphate_w } \\
\text { ater }\end{array}$ & abiotic_water & sPO4W \\
\hline DDetpoolW & detritus_DW_pool_water & abiotic_water & sDDetW \\
\hline NDetpoolW & detritus_N_pool_water & abiotic_water & sNDetW \\
\hline PDetpoolW & detritus_P_pool_water & abiotic_water & sPDetW \\
\hline O2poolw & oxygen_pool & abiotic_water & $\mathrm{sO} 2 \mathrm{~W}$ \\
\hline NH4pools & $\begin{array}{l}\text { uptake_target_Ammonium_s } \\
\text { ediment }\end{array}$ & abiotic_sediment & sNH4S \\
\hline NO3pools & $\begin{array}{l}\text { uptake_target_Nitrate_sedim } \\
\text { ent }\end{array}$ & abiotic_sediment & sNO3S \\
\hline PO4pools & $\begin{array}{l}\text { uptake_target_Phosphate_s } \\
\text { ediment }\end{array}$ & abiotic_sediment & sPO4S \\
\hline DDetpoolS & detritus_DW_pool_sediment & abiotic_sediment & sDDetS \\
\hline NDetpoolS & detritus_N_pool_sediment & abiotic_sediment & sNDetS \\
\hline PDetpoolS & detritus_P_pool_sediment & abiotic_sediment & sPDetS \\
\hline afOxySed & fraction_aerobic_sediment & abiotic_sediment & afOxySed \\
\hline
\end{tabular}

\section{S1.6 Zooplankton}

\section{File name:}


zooplankton.F90

\section{Module description:}

The zooplankton module describes zooplankton behavior in the water column.

The local processes are:

Zooplankton: assimilation, respiration (only for sDZoo), excretion (only for sNZoo, sPZoo), mortality and consumption by juvenile fish.

\section{List of variables (in following tables):}

Supplemental Table S17 List of local state variables in zooplankton module

\begin{tabular}{ll}
\hline State variable & Description of state variables \\
\hline SDZoo & zooplankton in water column, dry weight,mgDW/I \\
sPZoo & zooplankton in water column,phosphorus,mgP/l \\
sNZoo & zooplankton in water column,nitrogen,mgN/l
\end{tabular}

Supplemental Table S18 List of local diagnostic variables in zooplankton module

Diagnostic Description of diagnostic variable

variable

None None

Supplemental Table S19 List of dependencies in zooplankton module 


\begin{tabular}{|c|c|c|c|}
\hline $\begin{array}{l}\text { dependencie } \\
\mathrm{s}\end{array}$ & Description & Linked external module & $\begin{array}{l}\text { Linked external } \\
\text { variable }\end{array}$ \\
\hline uTm & temperature & physical driver & temperature \\
\hline par & $\begin{array}{l}\text { photosynthetically active } \\
\text { radiation }\end{array}$ & physical driver & $\begin{array}{l}\text { downwelling_photos } \\
\text { ynthetic_radiative_flu } \\
\text { x }\end{array}$ \\
\hline$d z$ & cell_thickness & physical driver & cell_thickness \\
\hline day & $\begin{array}{l}\text { number of days since } \\
\text { start of the year }\end{array}$ & physical driver & $\begin{array}{l}\text { number_of_days_sin } \\
\text { ce_start_of_the_year }\end{array}$ \\
\hline DfoodDiat & diatom_as_food_DW & phytoplankton_water & sDDiatW \\
\hline NfoodDiat & diatom_as_food_N & phytoplankton_water & sNDiatW \\
\hline PfoodDiat & diatom_as_food_P & phytoplankton_water & sPDiatW \\
\hline DfoodGren & green_as_food_DW & phytoplankton_water & sDGrenW \\
\hline NfoodGren & green_as_food_N & phytoplankton_water & sNGrenW \\
\hline PfoodGren & green_as_food_P & phytoplankton_water & sPGrenW \\
\hline DfoodBlue & blue_as_food_DW & phytoplankton_water & sDBlueW \\
\hline NfoodBlue & blue_as_food_N & phytoplankton_water & sNBlueW \\
\hline PfoodBlue & blue_as_food_P & phytoplankton_water & sPBluew \\
\hline DDetpoolW & detritus_as_food_DW & abiotic_water & sDDetW \\
\hline NDetpoolW & detritus_as_food_N & abiotic_water & sNDetW \\
\hline PDetpoolW & detritus_as_food_P & abiotic_water & sPDetW \\
\hline SiDetpoolW & detritus_as_food_si & abiotic_water & sSiDetW \\
\hline DfoodBent & benthos_as_food_DW & phytoplankton_sediment & sDBent \\
\hline NH4poolW & NH4_pool_for_web & abiotic_water & sNH4W \\
\hline NO3poolW & NO3_pool_for_web & abiotic_water & sNO3W \\
\hline PO4poolW & PO4_pool_for_web & abiotic_water & sPO4W \\
\hline aDSubVeg & submerged_macrophytes & macrophytes & aDSubVeg \\
\hline
\end{tabular}




\begin{tabular}{llll}
\hline dependencie & Description & Linked external module & Linked external \\
$\mathbf{s}$ & & & variable \\
\hline tDEnvFiAd & environmental correction & zoobenthos & tDEnvFiAd \\
aDSatFiAd & Saturation concentration & zoobenthos & aDSatFiAd \\
& of adult fish & & \\
& & & \\
\hline
\end{tabular}

\section{S1.7 Fish}

\section{File name:}

fish.F90

\section{Module description:}

Fish module describes zooplanktivorous fish, zoobenthivorous fish and piscivorous fish behavior in the water column. The local processes include:

zooplanktivorous and benthivorous fish (represented by juvenile and adults, respectively) and piscivorous fish.

Juvenile fish: migration, reproduction (fraction of adult fish biomass become juvenile fish), assimilation (predation on zooplankton), respiration (only for sDFiJv), excretion (only for PFiJv, sNFiJv), mortality, consumption by piscivorous fish and aging (part of the juvenile fish become adult fish)

Adult fish: migration, reproduction (fraction of adult fish biomass become juvenile fish), respiration (only for sDFiAd), excretion (only for sPFiAd and sNFiAd), mortality, consumption by piscivorous fish, and aging (part of juvenile fish become adult fish), (note: the assimilation of adult fish occurs in the benthic module, where adult fish predating the zoobenthos).

Piscivorous fish: migration, assimilation, respiration and mortality (piscivorous fish have fixed N/D and P/D ratio).

\section{List of variables (in following tables):}


Supplemental Table S20 List of local state variables in fish module

\begin{tabular}{|c|c|}
\hline State variable & Description of state variables \\
\hline sDFiJv & young fish in water,dry weight,mgDW/l \\
\hline SPFiJv & young fish in water,phosphorus, mgP/l \\
\hline sNFiJv & young fish in water,nitrogen,mgN/l \\
\hline sDFiAd & adult fish in water, dry weight,mgDW/l \\
\hline sPFiAd & adult fish in water, phosphorus,mgP/l \\
\hline sNFiAd & adult fish in water, nitrogen,mgN/l \\
\hline sDPisc & piscivorous fish in water, dry weight,mgDW// \\
\hline
\end{tabular}

Supplemental Table S21 List of local diagnostic variables in fish module

\begin{tabular}{ll}
\hline Diagnostic & Description of diagnostic variable \\
variable & \\
\hline aNPisc & piscivorous fish in nitrogen, $\mathrm{mgN} / \mathrm{l}$ \\
aPPisc & piscivorous fish in phosphorus, $\mathrm{mgP} / \mathrm{l}$ \\
\hline
\end{tabular}

Supplemental Table S22 List of dependencies in fish module

\begin{tabular}{llll}
\hline dependencie & Description & Linked external module & Linked external \\
s & & & variable \\
\hline uTm & temperature & physical driver & temperature \\
par & photosynthetically active & physical driver & downwelling_photos \\
& radiation & & ynthetic_radiative_flu \\
dz & cell_thickness & physical driver & x \\
day & number of days since & physical driver & cell_thickness \\
& start of the year & & number_of_days_sin \\
NH4poolW & NH4_pool_for_web & abiotic_water & ce_start_of_the_year \\
& & & sNH4W
\end{tabular}




\begin{tabular}{llll}
\hline dependencie & Description & Linked external module & Linked external \\
s & & & variable \\
\hline NO3poolW & NO3_pool_for_web & abiotic_water & sNO3W \\
PO4poolW & PO4_pool_for_web & abiotic_water & sPO4W \\
aDSubVeg & submerged_macrophytes & macrophytes & aDSubVeg \\
tDEnvFiAd & environmental correction & zoobenthos & tDEnvFiAd \\
aDSatFiAd & Saturation concentration & zoobenthos & aDSatFiAd \\
& of adult fish & &
\end{tabular}

\section{S1.8 Zoobenthos}

\section{File name:}

zoobenthos.F90

\section{Module description:}

The zoobenthos module describes the state variables relating to zoobenthos, thus sDBent, sPBent and sNBent. Local processes include consumption, migration, assimilation, and respiration (only for sDBent), excretion (only for sNBent and sPBent) and mortality. This module also describes the process of benthivorous fish (adult fish) predating on zoobenthos.

\section{List of variables (in following tables):}

Supplemental Table S23 List of local state variables in zoobenthos module

\begin{tabular}{ll}
\hline State variable & Description of state variables \\
\hline sDBent & zoobenthos, in dry weight,gDW/m*2 \\
sPBent & zoobenthos, in phosphorus, $g \mathrm{PP} / \mathrm{m}^{\star \star 2}$ \\
sNBent & zoobenthos, in nitrogen, $\mathrm{gN} / \mathrm{m}^{\star \star 2}$ \\
\hline
\end{tabular}

Supplemental Table S24 List of local diagnostic variables in zoobenthos module 
None

Supplemental Table S25 List of dependencies in zoobenthos module

\begin{tabular}{|c|c|c|c|}
\hline $\begin{array}{l}\text { dependencie } \\
\text { s }\end{array}$ & Description & Linked external module & $\begin{array}{l}\text { Linked external } \\
\text { variable }\end{array}$ \\
\hline uTm & temperature & physical driver & temperature \\
\hline sDepthw & water depth & physical driver & bottom_depth \\
\hline DDetpoolS & detritus_as_food_DW & abiotic_sediment & sDDetS \\
\hline PDetpoolS & detritus_as_food_P & abiotic_sediment & sPDetS \\
\hline NDetpoolS & detritus_as_food_N & abiotic_sediment & sNDetS \\
\hline SiDetpoolS & detritus_as_food_Si & abiotic_sediment & sSiDetS \\
\hline PO4pools & PO4_pool_web_sed & abiotic_sediment & sPO4S \\
\hline NH4pools & NH4_pool_web_sed & abiotic_sediment & sNH4S \\
\hline NO3pools & NO3_pool_web_sed & abiotic_sediment & sNO3S \\
\hline DfoodDiatS & diatom_as_food_DW & phytoplankton_sediment & sDDiatS \\
\hline PfoodDiatS & diatom_as_food_P & phytoplankton_sediment & sPDiatS \\
\hline NfoodDiatS & diatom_as_food_N & phytoplankton_sediment & sNDiatS \\
\hline DfoodGrens & green_as_food_DW & phytoplankton_sediment & sDGrenS \\
\hline PfoodGrens & green_as_food_P & phytoplankton_sediment & sPGrens \\
\hline NfoodGrens & green_as_food_N & phytoplankton_sediment & sNGrens \\
\hline DfoodBlues & blue_as_food_DW & phytoplankton_sediment & sDBlues \\
\hline PfoodBlues & blue_as_food_P & phytoplankton_sediment & sPBlues \\
\hline NfoodBlues & blue_as_food_N & phytoplankton_sediment & sNBlues \\
\hline DAdFish & adult_fish_DW & Fish & sDFiAd \\
\hline NAdFish & adult_fish_N & Fish & sNFiAd \\
\hline PAdFish & adult_fish_P & Fish & sPFiJv \\
\hline
\end{tabular}




\begin{tabular}{llll}
\hline dependencie & Description & Linked external module & Linked external \\
$\mathbf{s}$ & & & variable \\
\hline DJvFish & young_fish_DW & fish & sDFiJv \\
aCovVeg & macrophytes_coverage & macrophytes & aCovVeg \\
\hline
\end{tabular}

\section{S1.9 Auxiliary module}

File name:

auxiliary.F90

\section{Module description:}

The auxilary module is created for the purpose of computing resuspension and sedimentation, erosion and sediment burial processes (that influence several other modules).

\section{List of variables (in following tables):}

Supplemental Table S26 List of dependencies in auxiliary module dependencie Description Linked external module Linked external $\mathbf{s}$ variable

\begin{tabular}{llll}
\hline UTm & temperature & physical driver & $\begin{array}{l}\text { temperature } \\
\text { sDepthW }\end{array}$ \\
wWNH4 & ammonium pool in water depth & physical driver & bottom_depth \\
& column & sNH4W \\
SWNO3 & nitrate_pool_in_water & abiotic_water & sNO3W \\
SWPO4 & phosphate_pool_in_water & abiotic_water & sPO4W \\
SWPAIM & adsorbed_phosphorus_in_ & abiotic_water & sPAIMW \\
& water & & \\
SWDIM & inorg_pool_in_water & abiotic_water & sIMW
\end{tabular}




\begin{tabular}{|c|c|c|c|}
\hline $\begin{array}{l}\text { dependencie } \\
\text { s }\end{array}$ & Description & Linked external module & $\begin{array}{l}\text { Linked external } \\
\text { variable }\end{array}$ \\
\hline SWSiO2 & SiO2_release_to_water & abiotic_water & sSiO2W \\
\hline SWO2 & oxygen_pool & abiotic_water & $\mathrm{sO} 2 \mathrm{~W}$ \\
\hline SWDDet & detritus_DW_in_water & abiotic_water & sDDetW \\
\hline SWNDet & detritus_N_in_water & abiotic_water & sNDetW \\
\hline SWPDet & detritus_P_in_water & abiotic_water & sPDetW \\
\hline SWSiDet & detritus_Si_in_water & abiotic_water & sSiDetW \\
\hline SWDDiat & diatom_DW_in_water & phytoplankton_water & sDDiatW \\
\hline SWNDiat & diatom_N_in_water & phytoplankton_water & sNDiatW \\
\hline SWPDiat & diatom_P_in_water & phytoplankton_water & sPDiatW \\
\hline SWDGren & green_DW_in_water & phytoplankton_water & sDGrenW \\
\hline SWNGren & green_N_in_water & phytoplankton_water & sNGrenW \\
\hline SWPGren & green_P_in_water & phytoplankton_water & sPGrenW \\
\hline SWDBlue & blue_DW_in_water & phytoplankton_water & sDBluew \\
\hline SWNBlue & blue_N_in_water & phytoplankton_water & sNBluew \\
\hline SWPBlue & blue_P_in_water & phytoplankton_water & sPBluew \\
\hline WSDDiatS & diatom_DW_in_sediment & phytoplankton_sediment & sDDiatS \\
\hline WSNDiatS & diatom_N_in_sediment & phytoplankton_sediment & sNDiatS \\
\hline WSPDiatS & diatom_P_in_sediment & phytoplankton_sediment & sPDiatS \\
\hline WSDGrenS & green_DW_in_sediment & phytoplankton_sediment & sDGrens \\
\hline WSNGrenS & green_N_in_sediment & phytoplankton_sediment & sNGrens \\
\hline WSPGrenS & green_P_in_sediment & phytoplankton_sediment & sPGrens \\
\hline WSDBlues & blue_DW_in_sediment & phytoplankton_sediment & sDBlues \\
\hline WSNBlueS & blue_N_in_sediment & phytoplankton_sediment & sNBlues \\
\hline WSPBlueS & blue_P_in_sediment & phytoplankton_sediment & sPBlues \\
\hline DragVeg & vegetation_biomass & macrophytes & sDVeg \\
\hline
\end{tabular}




\begin{tabular}{llll}
\hline dependencie & Description & Linked external module & Linked external \\
$\mathbf{s}$ & & & variable \\
\hline TurbFish & adult_fish_biomass & fish & aDFish \\
tDAbioDetS & detritus_abiotic_update & zoobenthos & tDAbioDetS \\
tDAbioHumS & humus_abiotic_update & zoobenthos & \\
tDPrimDetS & detritus_from_algae & phytoplankton_sediment & tDPrimDetS \\
tDWebDetS & detritus_from_zoobenthos & zoobenthos & tDBenDetS \\
tDBedDetS & detritus_from_vegetation & macrophytes & tDBedDetS \\
\hline
\end{tabular}




\section{S2. List of FABM-PCLake biogeochemical sink and source terms}

This section provides a full list of the derivatives (and the processes in these) for the state variables implemented in FABM-PCLake. In order to distinguish the processes' modular location, different symbols are introduced to mark the processes (adopted from (Janse 2005)), see table S27 for the symbols representing the various modules.

Supplemental Table S27 Symbols of different FABM-PCLake module processes

\begin{tabular}{|c|c|c|}
\hline Modules & Module mark & Example \\
\hline abiotic water & () & (-wDMinDetW ) \\
\hline abiotic sediment & ( & (-tDMinDetS ) \\
\hline phytoplankton water & [ ] & [+ wDMortPhytW ] \\
\hline phytoplankton sediment & {[} & [+ tPMortPhytDetS] \\
\hline macrophytes & $\leq>$ & $\leq+$ tDMortVegW $>$ \\
\hline zooplankton & \{\} & $\{-$ wDConsDetZoo $\}$ \\
\hline fish & $<>$ & \\
\hline zoobenthos & \{\} & $\{-$ tDConsDetBent $\}$ \\
\hline auxiliary module & 0 & - tDSetIM + tDResusIM \\
\hline
\end{tabular}

\section{S 2.1 Abiotic water column module}

1) inorg. matter in water [mgD/l/s]

dDIMW $=$ - tDSetIM + tDResusIM+uDErosIMW

$=-$ settling + resuspension+erosion

2) detritus in water $[\mathrm{mgD} / / \mathrm{s}]$ 


\footnotetext{
$\mathrm{dDDetW}=-$ tDSetDet + tDResusDet $(-w D M i n D e t W)[+$ wDMortPhytW $] \leq+\mathrm{tDMortVegW}>$ $\{$-wDConsDetZoo + wDEgesZoo + wDMortZoo $\}<+$ tDEgesFish + tDMortFishDet +tDEgesPisc + tDMortPiscDet>
}

3) detritus in water [mgP/l/s]

$\mathrm{dPDetW}=-\mathrm{tPSetDet}+\mathrm{tPResusDet}(-\mathrm{wPMinDetW})[+$ wPMortPhytDetW $] \leq+$

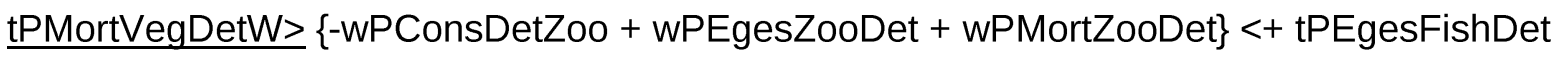
+ tPMortFishDet +tPEgesPiscDet + tPMortPiscDet>

4) detritus in water [mgN/l/s]

$\mathrm{dNDetW}=-$ tNSetDet + tNResusDet $(-$ wNMinDetW $)[+$ wNMortPhytDetW $] \leq+$ tNMortVegDetW $>\{$-wNConsDetZoo + wNEgesZooDet + wNMortZooDet $\}<+$ tNEgesFishDet + tNMortFishDet+ tNEgesPiscDet + tNMortPiscDet>

$=-$ settling + resuspension $(-$ mineralisation $)[+$ algal mortality $] \leq+$ part of macrophyte $\underline{\text { mortality in water }}\{$ \{- zooplankton detritus consumption +zooplankton egestion and mortality $\}$ $<+$ whitefish egestion and mortality + pred.fish egestion and mortality>

5) detritus [mgSi/ //s]

$\mathrm{dSiDetW}=-$ tSiSetDet + tSiResusDet $(-$ wSiMinDetW $)[+$ wSiMortDiatW $]\{+$ wSiConsDiatZoo\}

$=-$ settling + resuspension $(-$ mineralisation $)[+$ diatoms mortality] $\{+$ zooplankton diatoms consumption\}

6) $\mathrm{PO} 4$ in water $[\mathrm{mgP} / \mathrm{ls}]$

$\mathrm{dPO} 4 \mathrm{~W}=(\mathrm{wPMinDetW}-\mathrm{wPS}$ orpIMW + tPDifPO4) + tPResusPO4 [- wPUptPhyt + wPExcrPhytW +wPMortPhytPO4W] $\leq-$ tPUptVegW + tPExcrVegW + tPMortVegPO4W $>\{+$ 
wPExcrZoo + wPEgesZooPO4 + wPMortZooPO4\} <+tPExcrFiJv + tPExcrFiAd + tPEgesFishPO4 + tPMortFishPO4 + tPExcrPisc +tPEgesPiscPO4 + tPMortPiscPO4> $=($ mineralisation - sorption + diffusion from sediment $)+$ resuspension $[-$ algal uptake + algal excretion + part of algal mortality] $\leq-$ macrophyte uptake from water + macrophyte excretion in water + part of macrophyte mortality $\geq\{+$ zooplankton excretion and part of egestion and mortality $\}<+$ whitefish excretion and part of egestion and mortality + pred.fish excretion and part of egestion and mortality >

7) adsorbed $P$ in water [mgP/l/s]

dPAIMW $=$ tPResusAIM - tPSetAIM $(+$ wPSorpIMW)

$=$ resuspension - settling (+ sorption)

8) ammonium in water $[\mathrm{mgN} / \mathrm{l} / \mathrm{s}]$

$\mathrm{dNH} 4 \mathrm{~W}=(\mathrm{wNMinDetW}-\mathrm{wNNitrW}-\mathrm{tNDifNH} 4)+\mathrm{tNResusNH4}[-$ wNUptNH4Phyt + wNExcrPhytW + wNMortPhytNH4W] $\leq-$ tNUptNH4VegW + tNExcrVegW + tNMortVegNH4W $>$ $\{+$ wNExcrZoo + wNEgesZooNH4 +wNMortZooNH4 + tNExcrFiJv + tNExcrFiAd + tNEgesFishNH4 + tNMortFishNH4 + tNExcrPisc + tNEgesPiscNH4 + tNMortPiscNH4

$=($ mineralisation - nitrification in water - diffusion from sediment $)+$ resuspension $[-$ algal uptake + algal excretion + part of algal mortality - macrophyte uptake from water $] \leq+$ macrophyte excretion in water + part of macrophyte mortality $\geq\{+$ zooplankton excretion and part of egestion and mortality\} <+ whitefish excretion and part of egestion and mortality + pred.fish excretion and part of egestion and mortality>

9) nitrate in water [mgN///s]

dNO3W $=($ wNNitrW - wNDenitW + tNDifNO3) +tNResusNO3 [- wNUptNO3Phyt $] \leq-$ $\underline{\text { tNUptNO3VegW }>}$ 
$=($ nitrification in water - denitrif. in water + diffusion from sediment $)+$ resuspension $[-$ algal uptake] $\leq-$ macrophyte uptake $>$.

10) oxygen in water $[\mathrm{mgO} 2 / \mathrm{l} / \mathrm{s}]$

dO2W $=($ tO2Aer - wO2MinDetW - wO2NitrW $)(-$ tO2MinDetS + tO2NitrS $)[+$

wO2ProdPhyt - wO2RespPhytW +wO2UptNO3Phyt] $\leq+$ tO2ProdVegW - tO2RespVegW + $\underline{\text { tO2UptNO3VegW> }}$

$=($ reaeration - mineralisation - nitrification $)(-$ sediment oxygen demand $)[+$ algal production - algal respiration + nitrate uptake by algae] $\leq+$ macrophyte production $\underline{\text { macrophyte respiration + nitrate uptake by macrophytes }>}$

11) dissolved silicon dioxide in water [mgSi/ I/ s]

$\mathrm{dSiO} 2 \mathrm{~W}=($ wSiMinDetW $)(+(1.0-$ fRefrDetS $) *$ tSiMinDetS $)[-$ wSiUptDiat + wSiExcrDiatW] [+ tSiExcrDiatS]

$=($ mineralisation in water $)(+$ mineralisation in sediment $)[-$ diatoms uptake + diatoms excretion in water] [+ diatoms excretion in sed.]

\section{S2.2 Abiotic sediment module}

12) sediment inorg. matter $[\mathrm{gD} / \mathrm{m} 2 / \mathrm{s}]$

dDIMS = tDSetIM - tDResusIM - tDBurIM+uDErosIMS

$=$ settling - resuspension - burial+erosion

13) sediment detritus $[\mathrm{gD} / \mathrm{m} 2 / \mathrm{s}]$

dDDetS $=$ tDSetDet - tDResusDet - tDBurDet $(-$ tDMinDetS $)$ [+ tDMortPhytS]

$\leq+$ tDMortVegS $>\{-$ tDConsDetBent + tDEgesBent + tDMortBent $\}$

14) sediment detritus $P[\mathrm{gP} / \mathrm{m} 2 / \mathrm{s}]$ 
dPDetS $=$ tPSetDet - tPResusDet - tPBurDet (- tPMinDetS) $[+$ tPMortPhytDetS $] \leq+$ $\underline{\text { tPMortVegDetS }>\{- \text { tPConsDetBent }+ \text { tPEgesBent }+ \text { tPMortBent }\}}$

15) sediment detritus $\mathrm{N}[\mathrm{gN} / \mathrm{m} 2 / \mathrm{s}]$

dNDetS $=$ tNSetDet - tNResusDet - tNBurDet (- tNMinDetS) [+ tNMortPhytDetS] $\leq+$ $\underline{\text { tNMortVegDetS }>\{- \text { tNConsDetBent }+ \text { tNEgesBent }+ \text { tNMortBent }\}}$

$=$ settling - resuspension - burial $(-$ mineralisation $)[+$ sed. algal mortality $] \leq+$ macrophyte mortality in sed $>$ \{-zoobenthos detritus consumption + zoobenthos egestion and mortality\}

16) sediment detritus Si [gSi/m2/s]

dSiDetS = tSiSetDet - tSiResusDet - tSiBurDet (- tSiMinDetS) [+ tSiMortDiatS] $\{+$ tSiConsDiatBent $\}$

$=$ settling - resuspension - burial $(-$ mineralisation $)[+$ sed.diatoms mortality $[-$ zoobenthos diatoms consumption\}

17) phosphate in pore water $[\mathrm{gP} / \mathrm{m} 2 / \mathrm{s}]$

$\left.\mathrm{dPO} 4 \mathrm{~S}=\underline{(\mathrm{tPMinDetS}}{ }^{*}(1.0-\mathrm{fRefrDetS})-\mathrm{tPSorpIMS}-\mathrm{tPDifPO} 4-\mathrm{tPChemPO4}\right)-$ tPResusPO4 - tPBurPO4 [+ tPExcrPhytS + tPMortPhytPO4S] $\leq-\mathrm{tPUptVegS} \mathrm{+} \mathrm{tPExcrVegS}$ + tPMortVegPO4S $>\{+$ tPExcrBent +tPEgesBentPO4 + tPMortBentPO4 $\}$

$=($ detritus and humus mineralisation - sorption - diffusion to/from water - immobilisation $)$ - resuspension - burial [+excretion and part of mortality of sed. algae]<- macrophyte uptake from sediment +macrophyte excretion in sediment + part of macrophyte mortality $>\{+$ $\underline{\text { excretion and part of egestion and mortality of zoobenthos\} }}$

18) adsorbed $P$ in sediment $[\mathrm{gP} / \mathrm{m} 2 / \mathrm{s}]$

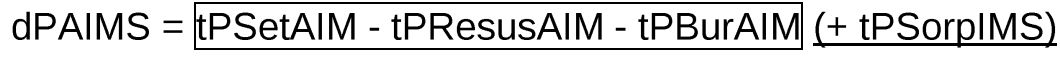

$=\underline{(\text { sorption })}+$ settling - resuspension - burial 
19) pore water ammonium $[\mathrm{gN} / \mathrm{m} 2 / \mathrm{s}]$

$\mathrm{dNH} 4 \mathrm{~S} \equiv($ tNMinDetS *(1.0-fRefrDetS $)-\mathrm{tNDifNH4}-\mathrm{tNNitrS})$-tNResusNH4- tNBurNH4 [+ tNExcrPhytS +tNMortPhytNH4S - tNUptNH4VegS] $\leq+$ tNExcrVegS + tNMortVegNH4S $>\{+$ $\underline{\text { tNExcrBent }+ \text { tNEgesBentNH4 + tNMortBentNH4\} }}$

$=$ (detritus and humus mineralisation - diffusion to/from water-nitrification in sediment) resuspension - burial [+excretion and part of mortality of sed. algae] <- macrophyte uptake from sediment + macrophyte excretion in sediment + part of macrophyte mortality $\geq\{+$ $\underline{\text { excretion and part of egestion and mortality of zoobenthos\} }}$

20) pore water nitrate $[\mathrm{gN} / \mathrm{m} 2 / \mathrm{s}]$

dNO3S $=($ tNNitrS - tNDenitS - tNDifNO3 $)-$ tNResusNO3 - tNBurNO3 $\leq-$ tNUptNO3VegS $>$

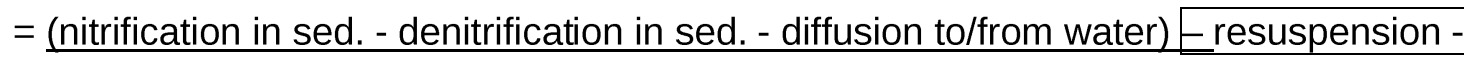

burial $\leq-$ macrophyte uptake from sediment $>$

21) Sediment humus $[\mathrm{gD} / \mathrm{m} 2 / \mathrm{s}]$

$\mathrm{dDHumS}=$ uDErosOM $-\mathrm{tDBurHum}(+$ fRefrDetS * tDMinDetS $-\mathrm{tDMinHumS})$

22) Sediment humus $[\mathrm{gN} / \mathrm{m} 2 / \mathrm{s}]$

$\mathrm{dNHumS}=$ uNErosOM - tNBurHum + (fRefrDetS * NDMinDetS - tNMinHumS

23) Sediment humus $[\mathrm{gP} / \mathrm{m} 2 / \mathrm{s}]$

dPHumS $=$ uPErosOM - tPBurHum $(+$ fRefrDetS * tPMinDetS - tPMinHumS $)$

$=$ erosion - burial $(+$ humification - mineralisation $)$ 


\section{S2.3 Phytoplankton water column module}

24) diatom dry weight in water column $[\mathrm{mgD} / / \mathrm{s}]$

$\mathrm{dDDiatW}=[\mathrm{wDAssDiat}-\mathrm{wDRespDiatW}-\mathrm{wDMortDiatW}]-\mathrm{tDSetDiat}+\mathrm{tDResusDiat}\{-$ wDConsDiatZoo\}

25) green algae dry weight in water column [mgD/l/s]

dDGrenW = [wDAssGren - wDRespGrenW - wDMortGrenW] - tDSetGren +

tDResusGren $\{-$ wDConsGrenZoo\}

26) cyanobacteria (blue-green algae) dry weight in water column [mgD/l/s]

dDBluew $=[$ wDAssBlue - wDRespBluew - wDMortBluew $]-$ tDSetBlue + tDResusBlue $\{-$ wDConsBlueZoo $\}$

$=[$ production - respiration - mortality $]-$ settling + resuspension $\{-$ grazing $\}$

27) diatom $P$ in water column $[\mathrm{mgP} / \mathrm{l} / \mathrm{s}]$

$\mathrm{dPDiatW}=[\mathrm{wPUptDiat}-\mathrm{wPExcrDiatW}-\mathrm{wPMortDiatW}]-$ tPSetDiat + tPResusDiat wPConsDiatZoo\}

28) green algae $P$ in water column $[\mathrm{mgP} / \mathrm{l} / \mathrm{s}]$

dPGrenW = [wPUptGren - wPExcrGrenW - wPMortGrenW] - tPSetGren + tPResusGren $\{-$ wPConsGrenZoo $\}$

29) cyanobacteria (blue-green algae) $\mathrm{P}$ in water column [mgP/l /s]

dPBlueW $=[$ wPUptBlue - wPExcrBlueW - wPMortBluew $]-$ tPSetBlue + tPResusBlue $\{-$ wPConsBlueZoo\}

$=[$ uptake - excretion - mortality $]-$ settling + resuspension $\{$ - grazing $\}$ 
30) diatom $\mathrm{N}$ in water column $[\mathrm{mgN} / \mathrm{l} / \mathrm{s}]$

$\mathrm{dNDiatW}=[\mathrm{wNUptDiat}-\mathrm{wNExcrDiatW}-\mathrm{wNMortDiatW}]-\mathrm{tNSetDiat}+\mathrm{tNResusDiat}\{-$ wNConsDiatZoo\}

31) green algae $\mathrm{N}$ in water column [mgN// /s]

dNGrenW $=[$ wNUptGren - wNExcrGrenW - wNMortGrenW] - tNSetGren +

tNResusGren $\{-$ wNConsGrenZoo $\}$

32) cyanobacteria (blue-green algae) $\mathrm{N}$ in water column [mgN/l /s]

dNBluew $=[$ wNUptBlue - wNExcrBluew - wNMortBluew $]-$ tNSetBlue + tNResusBlue $\{-$ wNConsBlueZoo\}

$=[$ uptake - excretion - mortality $]-$ settling + resuspension $\{$ - grazing $\}$

\section{S2.4 Phytoplankton sediment module}

33) sediment diatom dry weight [gD/m2/s]

$\mathrm{dDDiatS}=\mathrm{tDSetDiat}-\mathrm{tDResusDiat}$ [- tDMortDiatS $-\mathrm{tDRespDiatS}]\{-\mathrm{tDConsDiatBent}\}$

34) sediment green algae dry weight $[\mathrm{gD} / \mathrm{m} 2 / \mathrm{s}]$

dDGrens = tDSetGren - tDResusGren [- tDMortGrenS - tDRespGrenS] $\{-$

$\underline{\text { tDConsGrenBent\} }}$

35) sediment cyanobacteria (blue-green algae) dry weight $[\mathrm{gD} / \mathrm{m} 2 / \mathrm{s}]$

dDBlues $=$ tDSetBlue - tDResusBlue $[-$ tDMortBlues - tDRespBlues $]\{-$

$\underline{\text { tDConsBlueBent\} }}$

$=$ settling - resuspension $[-$ mortality - respiration $]\{-$ zoobenthos consumption $\}$ 
36) sediment diatom phosphorus $[\mathrm{gP} / \mathrm{m} 2 / \mathrm{s}]$

$\mathrm{dPDiatS}=\mathrm{tPSetDiat}-\mathrm{tPResusDiat}$ [- tPMortDiatS $-\mathrm{tPExcrDiatS}]\{-\mathrm{tPConsDiatBent}\}$

37) sediment green algae phosphorus $[\mathrm{gP} / \mathrm{m} 2 / \mathrm{s}]$

dPGrens $=$ tPSetGren - tPResusGren [- tPMortGrens - tPExcrGrenS] $\{-$

$\underline{\text { tPConsGrenBent\} }}$

38) sediment cyanobacteria (blue-green algae) phosphorus [gP/m2/s]

dPBlues = tPSetBlue - tPResusBlue $[-$ tPMortBlues - tPExcrBlues] $\{-$ tPConsBlueBent $\}$

$=$ settling - resuspension $[-$ mortality - excretion $]$ \{- zoobenthos consumption $\}$

39) sediment diatom nitrogen $[\mathrm{gN} / \mathrm{m} 2 / \mathrm{s}]$

$\mathrm{dNDiatS}=$ tNSetDiat $-\mathrm{tNResusDiat}[-\mathrm{tNMortDiatS}-\mathrm{tNExcrDiatS}]\{-\mathrm{t}$ ConsDiatBent $\}$

40) sediment green algae nitrogen $[\mathrm{gN} / \mathrm{m} 2 / \mathrm{s}]$

dNGrenS $=$ tNSetGren - tNResusGren [-tNMortGrenS - tNExcrGrenS] $\{$ -

tNConsGrenBent\}

41) sediment cyanobacteria (blue-green algae) nitrogen [gN/m2/s]

dNBlues $=$ tNSetBlue - tNResusBlue - tNMortBlues - tNExcrBlues - tNConsBlueBent

$=$ settling - resuspension $[-$ mortality - excretion $]$ \{-zoobenthos consumption $\}$

\section{S2.5 Macrophytes module}

42) macrophytes dry weight $[\mathrm{gD} / \mathrm{m} 2 / \mathrm{s}]$

$\mathrm{dDVeg}=\leq$ tDProdVeg $-\mathrm{tDRespVeg}-\mathrm{tDMortVeg}+\mathrm{tDMigrVeg}>$ 
$=\leq$ production - respiration - mortality + migrationz $>$

43) macrophytes phosphorus $[\mathrm{gP} / \mathrm{m} 2 / \mathrm{s}]$

$\mathrm{dPVeg}=\leq \mathrm{tPUptVeg}-\mathrm{tPExcrVeg}-\mathrm{tPMortVeg}+\mathrm{tPMigrVeg}>$

$=\leq$ uptake - excretion - mortality + migration $>$

44) macrophytes nitrogen [gN/m2/s]

$\mathrm{dNVeg}=\leq$ tNUptVeg - tNExcrVeg - tNMortVeg + tNMigrVeg $>$

$=\leq$ uptake - excretion - mortality + migration $>$

\section{S2.6 Zooplankton Module}

45) zooplankton dry weight $[\mathrm{mgD} / / \mathrm{s}]$

$\mathrm{dDZoo}=\{\mathrm{wDAssZoo}-\mathrm{wDRespZoo}-\mathrm{wDMortZoo}\}<-$ tDConsFiJv $>$

$=\{$ assimilation - respiration - mortality $\}<-$ fish predation $>$

46) zooplankton phosphorus [mgP/l/s]

$\mathrm{dPZoo}=\{\mathrm{wPAssZoo}-\mathrm{wPExcrZoo}-\mathrm{wPMortZoo}\}<-$ tPConsFiJv $>$

$=\{$ assimilation - excretion - mortality $\}<-$ fish predation $>$

47) zooplankton phosphorus [mgN///s]

$\mathrm{dNZoo}=\{\mathrm{wNAsSZoo}-\mathrm{wNExcrZoo}-\mathrm{wNMortZoo}-\mathrm{tNConsFiJv}\}$

$=\{$ assimilation - excretion - mortality $\}<-$ fish predation $>$

\section{S2.7 Fish module}

48) juvenile whitefish dry weight[mgD/l/s] 


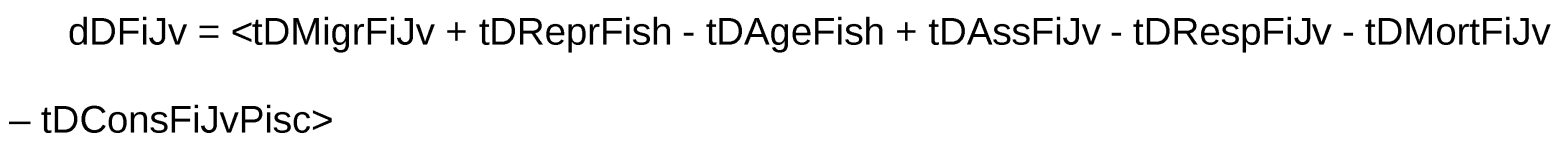

49) juvenile whitefish phosphorus [mgP///s]

$\mathrm{dPFiJv}=<\mathrm{tPMigrFiJv}+$ tPReprFish - tPAgeFish + tPAssFiJv - tPRespFiJv - tPMortFiJv tPConsFiJvPisc>

50) juvenile whitefish nitrogen [mgN/l/s]

$\mathrm{dDFiJv}=<\mathrm{tDMigrFiJv}+\mathrm{tDReprFish}-\mathrm{tDAgeFish}+\mathrm{tDAssFiJv}-\mathrm{tDRespFiJv}-\mathrm{tDMortFiJv}$ - tDConsFiJvPisc>

$=<$ migration + reproduction - ageing + assimilation - respiration - mortality -predation $>$

51) adult whitefish dry weight[mgD/l/s]

dDFiAd $=<$ tDMigrFiAd - tDReprFish + tDAgeFish - tDRespFiAd - tDMortFiAd tDConsFiAdPisc - tDHarvFish $>\underline{\{+ \text { tDAssFiAd }\}}$

52) adult whitefish phosphorus [mgP///s]

dPFiAd $=<$ tPMigrFiAd - tPReprFish + tPAgeFish - tPRespFiAd - tPMortFiAd tPConsFiAdPisc $>\{+$ tPAssFiAd $\}$

53) adult whitefish nitrogen $[\mathrm{mgN} / \mathrm{l} / \mathrm{s}]$

dNFiAd $=<$ tNMigrFiAd - tNReprFish + tNAgeFish - tNRespFiAd - tNMortFiAd tNConsFiAdPisc $>\underline{\{+ \text { tNAssFiAd }}$

$=<$ migration - reproduction + ageing - respiration - mortality - predation $>\{+$ assimilation $\}$

54) piscivorous fish dry weight[mgD/l/s]

$\mathrm{dDPisc}=\langle\mathrm{tDMigrPisc}+\mathrm{tDAssPisc}-\mathrm{tDRespPisc}-\mathrm{tDMortPisc}>$

$=<$ migration + assimilation - respiration - mortality $>$ 


\section{S2.8 Zoobenthos module}

55) zoobenthos dry weight[gD/m2/s]

$\mathrm{dDBent}=\{\mathrm{tDMigrBent}+\mathrm{tDAssBent}-\mathrm{tDRespBent}-\mathrm{tDM}$ ortBent $-\mathrm{tDConsFiAd}\}$

56) zoobenthos phosphorus $[\mathrm{gP} / \mathrm{m} 2 / \mathrm{s}]$

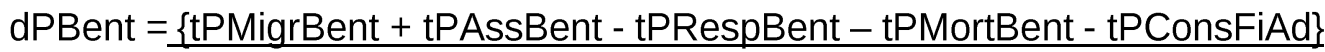

57) zoobenthos nitrogen[gN/m2/s]

$\mathrm{dNBent}=\{$ tNMigrBent + tNAssBent $-\mathrm{tNRespBent}-\mathrm{tNMortBent}-\mathrm{tNConsFiAd}\}$

$=\{$ migration + assimilation - respiration - mortality - fish predation $\}$ 
S3. FABM-PCLake parameter list (input file in .yaml format) in modular structure

The input file can be downloaded from www.fabm.net and use of the template provided here is recommended as a basis for parameter adaptation, as the yaml file format includes a strict indentation standard.

\section{S3.1 FABM-PCLake_abiotic_water}

model: pclake_abiotic_water

long_name: abiotic_process_in_water

initialization:

sNH4W: $0.1 \quad \# \mathrm{mgN} / \mathrm{l}$, ammonium in water

sNO3W: $0.1 \quad \# \mathrm{mgN} / \mathrm{l}$, nitrite in water

sPO4W: 0.01 \#mgP/l, phosphate in water

SPAIMW: 0.0 \# mgP/l, adsorbed phosphorus on inorg. matter in water

sSiO2W: 3.0 \# mgSi/l, dissolved silicon dioxide in water

sO2W: 10.0 \# mgO2/l, oxygen in water

sDIMW: 5.0 \# mgDW/l, inorg.matter in water

sDDetW: 2.0 \# mgDW/I, water detritus in dry weight

sPDetW: $0.005 \quad$ \# mgP/l, water detritus in phosphorus

sNDetW: 0.05 \# mgN/l, water detritus in nitrogen

sSiDetW: 0.02 \# mgSi/l, water detritus in silica

parameters:

cCPerDW: $0.4 \#$ gC/gDW, C content of organic matter

cExtSpDet: $0.15 \quad$ \# m2/gDW,specific_extinction_detritus

cExtSpIM: $0.05 \quad \# 0.05 \mathrm{~m} 2 / \mathrm{gDW}$ specific_extinction_inert_matter

cExtWat: 0.5 \# background_extinction"

cKPAdsOx: $0.6 \quad \# \mathrm{~m} 3 / \mathrm{gP}, \mathrm{P}$ adsorption affinity at oxidized conditions

cRelPAdsAl: $0.134 \quad$ \# gP/gAl, max. P adsorption per g Al

cRelPAdsD: 0.00003 \# gP/gD, max. P adsorption per g DW 
cRelPAdsFe: 0.065 \# gP/gFe, max. P adsorption per g Fe

cThetaAer: $1.024 \quad \# 1 / \mathrm{e}^{\wedge} \mathrm{oC}$, temperature coeff. for reaeration

cThetaMinW: 1.07 \# exponantional temperature constant of mineralisation in water

cThetaNitr: 1.08 \# temperature coefficient for nitrification

fAIDIM: $0.01 \quad \# \mathrm{gAl} / \mathrm{gD}$, Al content of inorg. matter

fFeDIM: 0.01 \# gFe/gD, Fe content of inorg. matter

fRedMax: 0.9 \# max. reduction factor of $P$ adsorption affinity

hNO3Denit: 2 \# mgN/l, quadratic half-sat. NO3 conc. for denitrification

hO2BOD: 1 \# mgO2/l, half-sat. oxygen conc. for BOD

hO2Nitr: 2 \# mgO2/l ,half-sat_O2_conc_for_nitrification_in_water

kDMinDetW: 0.01 \# day-1, decomposition constant of detritus

kNitrW: 0.1 \# day-1, nitrification rate constant in water

kPSorp: 0.05 \# day-1, P sorption rate constant not too high -> model speed day

NO3PerC: $0.8 \quad$ \# mol NO3 denitrified per mol C mineralised

O2PerNH4: $2 \quad$ \# mol O2 used per mol NH4+ nitrified

\section{\# Sinking}

cVSetIM: -1.0 \# m/day, max. sedimentation velocity of inert org. matter

cVSetDet: $-0.25 \quad \# \mathrm{~m} /$ day, max. sedimentation velocity of detritus

\section{S3.2 FABM-PCLake_abiotic_sediment}

model: pclake_abiotic_sediment

long_name: abiotic_process_in_sediment

initialization:

sNH4S: $0.02 \quad \# \mathrm{gN} / \mathrm{m} 2$, dissolved $\mathrm{N}-\mathrm{NH} 4$ in interstitial water

sNO3S: $0.002 \quad \# \mathrm{gN} / \mathrm{m} 2$, dissolved N-NO3 in interstitial water

sPO4S: $0.181703 \quad \# \mathrm{gP} / \mathrm{m} 2$, dissolved P-PO4 in interstitial water

SPAIMS: $17.9886 \quad \# \mathrm{gP} / \mathrm{m} 2$, adsorbed $\mathrm{P}$ in sediment

sDIMS: $32706.5 \quad$ \# gDW/m2; Inorg matter in sediment 
sDDetS: $181.703 \quad \#$ gDW/m2, detritus in sediment

sNDetS: $4.54258 \quad \# \mathrm{gN} / \mathrm{m} 2$, detritus in sediment

sPDetS: $0.454258 \quad \# \mathrm{gP} / \mathrm{m} 2$, detritus in sediment

sSiDetS: $1.81703 \quad$ \# gSi/m2, detritus in sediment

sDHumS: $3452.36 \quad \# \mathrm{gDW} / \mathrm{m} 2$, humus in sediment,

sNHumS: $172.618 \quad \# \mathrm{gN} / \mathrm{m} 2$, humus in sediment,initial $\mathrm{N}$ fraction in humus

sPHumS: $17.2618 \quad \# \mathrm{gP} / \mathrm{m} 2$, humus in sediment, initial $P$ fraction in humus

parameters:

bPorS: $0.847947 \quad$ \# porosity [m3 water m-3 sediment

bPorCorS: 0.737275 \# sediment porosity, corrected for tortuosity

cCPerDW: $0.4 \quad \#$ gC/gDW, C content of organic matter

cDepthS: 0.1 \# sediment depth [m]

cKPAdsOx: $0.6 \quad \# \quad m 3 / g P, P$ adsorption affinity at oxidized conditions

coPO4Max: $1.0 \quad$ \# mgP/l, max. PO4 conc. in pore water

cRelPAdsAl: 0.134 \# gP/gAl, max. P adsorption per g Al

cRelPAdsD: 0.00003 \# gP/gD, max. P adsorption per g DW

cRelPAdsFe: 0.065 \# gP/gFe, max. P adsorption per g Fe

cThetaDif: 1.02 \# Temperature coefficient for diffusion

cThetaMinS: $1.07 \quad \#$-, exponantial Temperature constant of sediment mineralisation

cThetaNitr: 1.08 \# Temperature coefficient for nitrification

cTurbDifNut: 5.0 \# bioturbation factor for diffusion

cTurbDifO2: $5.0 \quad \#$-, bioturbation factor for diffusion

fAIDIM: $0.01 \quad$ \# gAl/gD, Al content of inorg. matter

fDepthDifS: 0.5 \# utrient diffusion distance as fraction of sediment depth

fFeDIM: $0.01 \quad \#$ gFe/gD, Fe content of inorg. matter

fRedMax: $0.9 \quad$ \# max. reduction factor of $P$ adsorption affinity

fRefrDetS: $0.15 \quad \#-$, refractory fraction of sed. detritus

hNO3Denit: $2.0 \quad$ \# mgN/l, quadratic half-sat. NO3 conc. for denitrification 
kDMinDetS: 0.002 \#"day-1, decomposition constant of sediment detritus

kNDifNH4: 0.000112 \# m2/day, mol. NH4 diffusion constant

kNDifNO3: 0.000086 \# m2/day, mol. NO3 diffusion constant

kNitrS: $1.0 \quad$ \# day-1,nitrification rate constant in sediment

kO2Dif: 0.000026 \# m2/day, mol. O2 diffusion constant

kPChemPO4: 0.03 \# day-1, chem. PO4 loss rate

kPDifPO4: 0.000072 \# m2/day, mol. PO4 diffusion constant

kPSorp: $0.05 \quad \#$ day-1, P sorption rate constant not too high $->$ model speed

NO3PerC: $0.8 \quad$ \# mol NO3 denitrified per mol C mineralised

O2PerNH4: $2.0 \quad \#$ mol O2 used per mol NH4+ nitrified

kDMinHum: 0.00001 \# day-1,maximum_decomposition_constant_of_humic_material_(1D-5)

coupling:

oxygen_pool_water: abiotic_water/sO2W

SiO2_generated_by_mineralisation: abiotic_water/sSiO2W

NH4_diffusion_flux: abiotic_water/sNH4W

NO3_diffusion_flux: abiotic_water/sNO3W

PO4_diffusion_flux: abiotic_water/sPO4W

\section{S3.3 FABM-PCLake_phytoplankton_water}

model: pclake_phytoplankton_water

long_name: phytoplankton_in_water

initialization:

sDDiatW: 0.5 \# diatoms_DW_in_lake_water

SPDiatW: 0.005 \# diatoms_P_in_lake_water

sNDiatW: 0.05 \# diatoms_N_in_lake_water

sDGrenW: 0.5 \# green_algae_DW_in_lake_water

SPGrenW: 0.005 \# green_algae_P_in_lake_water 
sNGrenW: 0.05 \# green_algae_N_in_lake_water

sDBlueW: 3.0 \# blue-greens_DW_in_lake_water

sPBlueW: 0.03 \# blue-greens_P_in_lake_water

sNBlueW: 0.3 \# blue-greens_N_in_lake_water

parameters:

cAffNUptBlue: $0.2 \quad \#$ I/mgDW/day, initial $\mathrm{N}$ uptake affinity bluegreens

cAffNUptDiat: $0.2 \quad \#$ I/mgDW/day, initial $\mathrm{N}$ uptake affinity diatoms

cAffNUptGren: 0.2 \# l/mgDW/day, initial N uptake affinity greens:

cAffPUptBlue: $0.8 \quad$ \# I/mgDW/day, initial P uptake affinity bluegreens

cAffPUptDiat: 0.2 \# I/mgDW/day, initial P uptake affinity diatoms

cAffPUptGren: $0.2 \quad$ \# //mgDW/day, initial P uptake affinity greens

cChDBlueMax: 0.015 \# mgChl/mgDW,max._chlorophyll/C_ratio_bluegreens

cChDBlueMin: 0.005 \# mgChl/mgDW,min._chlorophyll/C_ratio_bluegreens

cChDDiatMax: 0.012 \# mgChl/mgDW, max._chlorophyll/C_ratio_diatoms

cChDDiatMin: 0.004 \# mgChl/mgDW ,min._chlorophyll/C_ratio_diatoms

cChDGrenMax: 0.02 \# mgChl/mgDW,max._chlorophyll/C_ratio_greens

cChDGrenMin: 0.01 \# mgChl/mgDW, min._chlorophyll/C_ratio_greens

cCPerDW: $0.4 \quad \#$ gC/gDW, C content of organic

cExtSpBlue : $0.35 \quad \# 0.35 \mathrm{~m} 2 / \mathrm{gDW}$,specific_extinction_bluegreens

cExtSpDiat : 0.25 \# 0.25m2/gDW,specific_extinction_diatoms

cExtSpGren : 0.25 \# 0.25m2/gDW,specific_extinction_greens

cLOptRefBlue: $13.6 \quad \# \mathrm{~W} / \mathrm{m} 2$, optimum PAR for blue-greens at $20^{\circ} \mathrm{C}$ (Steele function)

cLOptRefDiat: 54.0 \# W/m2, optimum PAR for diatoms at $20^{\circ} \mathrm{C}$ (Steele function)

cMuMaxBlue: 0.6 \# day-1,maximum_growth_rate_bluegreens'

cMuMaxDiat: 2.0 \# day-1,maximum_growth_rate_diatoms

cMuMaxGren: 1.5 \# day-1,maximum_growth_rate_greens

cNDBlueMax: $0.15 \quad \# \mathrm{mgN} / \mathrm{mgDW}$, max. N/day ratio bluegreens

cNDBlueMin: $0.03 \quad \# \mathrm{mgN} / \mathrm{mgDW}$, minimum N/day ratio bluegreens 
cNDDiatMax: $0.05 \quad \#$ mgN/mgDW, max. N/day ratio diatoms

cNDDiatMin: $0.01 \quad \# \mathrm{mgN} / \mathrm{mgDW}$, minimum N/day ratio diatoms

cNDGrenMax: $0.1 \quad \#$ mgN/mgDW, max. N/day ratio greens

cNDGrenMin: $0.02 \quad \# \mathrm{mgN} / \mathrm{mgDW}$, minimum N/day ratio greens

cPDBlueMax: $0.025 \quad \# \mathrm{mgP} / \mathrm{mgDW}$, max. P/day ratio blue-greens

cPDBlueMin: 0.0025 \# mgP/mgDW, minimum P/day ratio bluegreens

cPDDiatMax: 0.005 \# mgP/mgDW, max. P/day ratio diatoms

cPDDiatMin: 0.0005 \# mgP/mgDW, minimum P/day ratio diatoms

cPDGrenMax: 0.015 \# mgP/mgDW, max. P/day ratio greens

cPDGrenMin: 0.0015 \# mgP/mgDW, minimum P/day ratio greens

cSiDDiat: $0.15 \quad$ \# mgSi/mgDW, Si/D ratio of diatoms

cSigTmBlue: $12.0 \quad \#{ }^{\circ} \mathrm{C}$, temperature constant bluegreens (sigma in Gaussian curve)

cSigTmDiat: $20.0 \quad \#^{\circ} \mathrm{C}$, temperature constant diatoms (sigma in Gaussian curve)

cSigTmGren: $15.0 \quad \#^{\circ} \mathrm{C}$, temperature constant greens (sigma in Gaussian curve)

cTmOptBlue: $25.0 \quad \#{ }^{\circ} \mathrm{C}$, optimum temperature bluegreens

cTmOptDiat: $18.0 \quad \#{ }^{\circ} \mathrm{C}$, optimum temperature diatoms

cTmOptGren: $25.0 \quad \#{ }^{\circ} \mathrm{C}$, optimum temperature of greens

cVNUptMaxBlue: 0.07 \# mgN/mgDW/day, maximum $\mathrm{N}$ uptake capacity of bluegreens cVNUptMaxDiat: 0.07 \# mgN/mgDW/day, maximum $\mathrm{N}$ uptake capacity of diatoms cVNUptMaxGren: 0.07 \# mgN/mgDW/day, maximum $\mathrm{N}$ uptake capacity of greens cVPUptMaxBlue: 0.04 \# mgP/mgDW/day, maximum P uptake capacity of bluegreens cVPUptMaxDiat: 0.01 \# mgP/mgDW/day, maximum P uptake capacity of diatoms cVPUptMaxGren: 0.01 \# mgP/mgDW/day, maximum P uptake capacity of greens fDissMortPhyt: 0.2 \#[-], soluble_nutrient_fraction_of_died_ hLRefGren: $17.0 \quad \# \mathrm{~W} / \mathrm{m} 2$, half-sat. PAR for greens at $20^{\circ} \mathrm{C}$ (Lehmann function) hO2BOD: $1.0 \quad \#$ mgO2/l, half-sat. oxygen conc. for BOD hSiAssBlue: $0.0 \quad$ \# mgSi/l, half-sat. Si conc. for growth of bluegreens: 0 hSiAssDiat: 0.09 \# mgSi/l, half-sat. Si for diatoms 
hSiAssGren: 0.0 \# mgSi/l, half-sat. Si conc. for growth of green algae: 0

kDRespBlue: 0.03 \# day-1, maintenance respiration constant bluegreens (: 0.05 * MuMax)

kDRespDiat: 0.1 \# day-1, maintenance respiration constant diatoms (: 0.05 * MuMax)

kDRespGren: 0.075 \# day-1, maintenance respiration constant greens (: 0.05 * MuMax)

kMortBlueW: $0.01 \quad$ \# day-1, mortality constant of bluegreens in water

kMortDiatW: 0.01 \# day-1, mortality constant of diatoms in water

kMortGrenW: 0.01 \# day-1, mortality constant of diatoms in water

\# sinking

cVSetDiat: -0.5 \# m/day, sedimentation velocity diatoms

cVSetGren: -0.2 \# m/day, sedimentation velocity of greens

cVSetBlue: -0.06 \# m/day, sedimentation velocity bluegreens

coupling:

SiO2_pool_water: abiotic_water/sSiO2W

sPO4_pool_water: abiotic_water/sPO4W

sNH4_pool_water: abiotic_water/sNH4W

sNO3_pool_water: abiotic_water/sNO3W

oxygen_pool_water: abiotic_water/sO2W

detritus_DW_pool_water: abiotic_water/sDDetW

detritus_N_pool_water: abiotic_water/sNDetW

detritus_P_pool_water: abiotic_water/sPDetW

detritus_Si_pool_water: abiotic_water/sSiDetW

surface_vegetation_coverage: macrophytes/afCovSurfVeg

\section{S3.4 FABM-PCLake_phytoplankton_sediment}

model: pclake_phytoplankton_sediment

long_name: phytoplankton_in_sediment

initialization:

sDDiatS: 0.001 \# diatoms_DW_on_lake_sediment 
SPDiatS: 0.00001 \# diatoms_P_on_lake_sediment

sNDiatS: 0.0001 \# diatoms_N_on_lake_sediment

sDGrenS: 0.001 \# green_algae_DW_on_lake_sediment

SPGrenS: 0.00001 \# green_algae_P_on_lake_sediment

sNGrenS: 0.0001 \# green_algae_N_on_lake_sediment

sDBlues: 0.001 \# bluegreens_DW_on_lake_sediment

sPBlueS: 0.00001 \# bluegreens_P_on_lake_sediment

sNBlueS: 0.0001 \# bluegreens_N_on_lake_sediment

parameters:

cNDBlueMax: $0.15 \quad \# \mathrm{mgN} / \mathrm{mgDW}$, max. N/day ratio bluegreens

cNDBlueMin: $0.03 \quad \# \mathrm{mgN} / \mathrm{mgDW}$, minimum N/day ratio bluegreens

cNDDiatMax: $0.05 \quad \# \mathrm{mgN} / \mathrm{mgDW}$, max. N/day ratio diatoms

cNDDiatMin: $0.01 \quad \# \mathrm{mgN} / \mathrm{mgDW}$, minimum N/day ratio diatoms

cNDGrenMax: $0.1 \quad \#$ mgN/mgDW, max. N/day ratio greens

cNDGrenMin: $0.02 \quad \# \mathrm{mgN} / \mathrm{mgDW}$, minimum N/day ratio greens

cPDBlueMax: 0.025 \# mgP/mgDW, max. P/day ratio bluegreens

cPDBlueMin: 0.0025 \# mgP/mgDW, minimum P/day ratio bluegreens

cPDDiatMax: $0.005 \quad \# \mathrm{mgP} / \mathrm{mgDW}$, max. P/day ratio diatoms

cPDDiatMin: 0.0005 \# mgP/mgDW, minimum P/day ratio diatoms

cPDGrenMax: 0.015 \# mgP/mgDW, max. P/day ratio greens

cPDGrenMin: 0.0015 \# mgP/mgDW, minimum P/day ratio greens

cSiDDiat: $0.15 \quad$ \# mgSi/mgDW, Si/D ratio of diatoms

cSigTmBlue: $12.0 \quad \#^{\circ} \mathrm{C}$, temperature constant bluegreens (sigma in Gaussian curve)

cSigTmDiat: $20.0 \quad \#{ }^{\circ} \mathrm{C}$, temperature constant diatoms (sigma in Gaussian curve)

cSigTmGren: $15.0 \quad \#{ }^{\circ} \mathrm{C}$, temperature constant greens (sigma in Gaussian curve)

cTmOptBlue: $25.0 \quad \#^{\circ} \mathrm{C}$, optimum temperature bluegreens

cTmOptDiat: $18.0 \quad \#^{\circ} \mathrm{C}$, optimum temperature diatoms

cTmOptGren: $25.0 \quad \#{ }^{\circ} \mathrm{C}$, optimum temperature of greens 
fDissMortPhyt: $0.2 \quad$ \#-, soluble nutrient fraction of dead algae

kDRespBlue: 0.03 \# day-1, maintenance respiration constant gluegreens (: 0.05 * MuMax)

kDRespDiat: $0.10 \quad$ \# day-1, maintenance respiration constant diatoms (: 0.05 * MuMax)

kDRespGren: 0.075 \# day-1, maintenance respiration constant greens (: 0.05 * MuMax)

kMortBlueS: $0.2 \quad$ \# day-1, mortality constant bluegreens

kMortDiatS: $0.05 \quad$ \# day-1, mortality constant of sed. diatoms

kMortGrenS: $0.05 \quad$ \# day-1, mortality constant greens

coupling:

sPO4_pool_sediment: abiotic_sediment/sPO4S

sNH4_pool_sediment: abiotic_sediment/sNH4S

sNO3_pool_sediment: abiotic_sediment/sNO3S

detritus_DW_pool_sediment: abiotic_sediment/sDDetS

detritus_N_pool_sediment: abiotic_sediment/sNDetS

detritus_P_pool_sediment: abiotic_sediment/sPDetS

detritus_Si_pool_sediment: abiotic_sediment/sSiDetS

SiO2_pool_water: abiotic_water/sSiO2W

\section{S3.5 FABM-PCLake_macrophytes}

model: pclake_macrophytes

long_name: macrophytes

initialization:

sDVeg: 1.0 \# vegetation_DW_in_lake_water

sNVeg: 0.02 \# vegetation_N_in_lake_water

SPVeg: 0.002 \# vegetation_P_in_lake_water

parameters:

bPorS: $0.847947 \quad$ \# porosity [m3 water m-3 sediment]

cAffNUptVeg: $0.2 \quad$ \# I/mgDW/day,initial_N_uptake_affinity_vegetation

cAffPUptVeg: $0.2 \quad$ \# I/mgDW/day,initial_P_uptake_affinity_vegetation 
cCovSpVeg: $0.5 \quad$ \# \%_cover/gDW/m2 specific_cover

cCPerDW: $0.4 \quad \#$ gC/gDW, C content of organic matte

cDayWinVeg: 259.0 \# day, end of growing season: 16 Sep

cDCarrVeg: $400.0 \quad$ \# gDW/m2, max. vegetation standing crop

cDepthS: $0.1 \quad$ \# sediment depth [m]

cDLayerVeg: $0.0 \quad \# \mathrm{gD} / \mathrm{m} 2$, biomass of a single layer floating leaves

cDVegln: $1.0 \quad$ \# gD/m2, external vegetation density

cExtSpVeg : $0.01 \quad \# 0.01 \mathrm{~m} 2 / \mathrm{gDW}$,specific_extinction

CLengAllo: $15.0 \quad$ \# day, duration of allocation and reallocation phase

CLengMort: $15.0 \quad$ \# day, length of shoot mortality period

cMuMaxVeg: $0.2 \quad \# \mathrm{~g} / \mathrm{g}$ shoot/day, maximum growth rate of vegetation at $20^{\circ} \mathrm{C}$

cNDVeg0: $0.02 \quad \# \mathrm{gN} / \mathrm{gD}$, initial $\mathrm{N}$ fraction in vegetation

cNDVegMax: $0.035 \quad \# \mathrm{mgN} / \mathrm{mgD}$, maximum $\mathrm{N} /$ day ratio vegetation

cNDVegMin: $0.01 \quad \# \mathrm{mgN} / \mathrm{mgD}$, minimum $\mathrm{N} /$ day ratio vegetation

cPDVeg0: $0.002 \quad \# \mathrm{gP} / \mathrm{gD}$, initial $\mathrm{P}$ fraction in vegetation

cPDVegMax: 0.0035 \# mgP/mgD, maximum P/day ratio vegetation

cPDVegMin: $0.0008 \quad \# \mathrm{mgP} / \mathrm{mg}$, minimum P/day ratio vegetation

cQ10ProdVeg: 1.2 \# temperature quotient of production

cQ10RespVeg: $2.0 \quad$ \# temperature quotient of respiration

cTmInitVeg: $9.0 \quad$ \# oC, temperature for initial growth

cVNUptMaxVeg: 0.1 \# mgN/mgDW/day, maximum_N_uptake_capacity_of_vegetation

cVPUptMaxVeg: 0.01 \# mgP/mgDW/day,maximum_P_uptake_capacity_of_vegetation

fDepth1Veg: 0.5

\#max._upper_depth_of_submerged_veget._layer,_as_fraction_of_water_depth

fDepth2Veg: $1.0 \quad$ \#

max._lower_depth_of_submerged_veget._layer,_as_fraction_of_water_depth

fDetWMortVeg: 0.1 \# fraction_of_shoot_mortality_becoming_water_detritus

fDissMortVeg: 0.25 \# fraction_dissolved_nutrients_from_died_plants 
fEmergVeg: $0.0 \quad$ \# g floating / g shoot, emergent fraction of shoot

fFloatVeg: $0.0 \quad$ \# g floating / $g$ shoot, floating fraction of shoot

fRootVegSum: $0.1 \quad$ \# g root / g veg, root fraction outside growing season

fRootVegWin: $0.6 \quad$ \# g root / g veg, root fraction outside growing season

fSedUptVegCoef: 2.66 \# sigm._regr._coeff._for_sediment_fraction_of_nutrient_uptake fSedUptVegExp : -0.83 \#

exponent_in_sigm._regr._for_sediment_fraction_of_nutrient_uptake

fSedUptVegMax: 0.998 \# maximum_sediment_fraction_of_nutrient_uptake

fWinVeg: $0.3 \quad$ \# fraction surviving in winter

hLRefVeg: $17.0 \quad$ \# W/m2 PAR, half-sat. light at $20^{\circ} \mathrm{C}$

hO2BOD: $1.0 \quad \#$ mgO2/l, half-sat. oxygen conc. for BOD

kDRespVeg: $0.02 \quad$ \# day-1, dark respiration rate of vegetation

kMigrVeg: $0.001 \quad$ \# day-1, vegetation migration rate

kMortVegSum: 0.005 \# day-1, vegetation mortality rate in Spring and Summer

UseEmpUpt: 0 \# 0=do_not_use_this_empirical_relation

coupling:

ammonium_pool_water: abiotic_water/sNH4W

nitrate_pool_water: abiotic_water/sNO3W

phosphate_pool_water: abiotic_water/sPO4W

ammonium_pool_sediment: abiotic_sediment/sNH4S

nitrate_pool_sediment: abiotic_sediment/sNO3S

phosphate_pool_sediment: abiotic_sediment/sPO4S

oxygen_pool_water: abiotic_water/sO2W

detritus_DW_pool_water: abiotic_water/sDDetW

detritus_N_pool_water: abiotic_water/sPDetW

detritus_P_pool_water: abiotic_water/sNDetW

detritus_DW_pool_sediment: abiotic_sediment/sDDetS

detritus_N_pool_sediment: abiotic_sediment/sNDetS 
detritus_P_pool_sediment: abiotic_sediment/sPDetS

oxic_layer_value: abiotic_sediment/afOxySed

\section{S3.6 FABM-PCLake_zooplankton}

model: zooplankton

long_name: zooplankton

initialization:

sDZoo: 0.05 \#zooplankton DW in water ( $\mathrm{g} \mathrm{m}$-3)

sPZoo: 0.0005 \#zooplankton P in water ( $\mathrm{g} \mathrm{m}$-3)

sNZoo: $0.0035 \quad$ \# zooplankton $\mathrm{N}$ in water ( $\mathrm{g} \mathrm{m}$-3)

parameters:

cSigTmZoo: 13.0 \# temperature constant zooplankton(sigma in Gaussian curve) ( C),

default $=13.0$

cTmOptZoo: $25.0 \quad$ \# optimum temp. zooplankton $(\diamond \mathrm{C})$, default $=25.0$

cDCarrZoo: 25.0 \# carrying capacity of zooplankton (gm-3), default = 25.0

kMortZoo: $0.04 \quad \#$ mortality_constant_herb.zooplankton (d-1), default $=0.04$

kDRespZoo: 0.15 \# maintenance respiration constant herb.zooplankton (d-1), default= 0.15

cPrefDiat: 0.75 \# selection factor for Diatoms $([-])$, default $=0.75$

cPrefGren: $0.75 \quad$ \# selection factor for Greens $([-])$, default $=0.75$

cPrefBlue: 0.125 \# selection factor for Bluegreens Cal. $([-])$, default $=0.125$

cPrefDet: $0.25 \quad$ \# selection factor for detritus ([-]), default $=0.25$

hFilt: $1.0 \quad$ \# half-sat. food conc. for filtering (gDW m-3), default $=1.0$

fDAssZoo: $0.35 \quad$ \# DW-assimilation efficiency of herb. zooplankton ([-]), default $=0.35$

cFiltMax: $4.5 \quad$ \# maximum filtering rate (Itr/mgDW/d), default $=4.5$

cPDZooRef: 0.01 \# reference P/C-ratio herb. zooplankton $(\mathrm{mgP} / \mathrm{mgDW})$, default $=0.01$ cNDZooRef: 0.07 \# reference N/C-ratio herb. zooplankton (mgN/mgDW), default $=0.07$ 
fDissEgesZoo: 0.25 \# oluble_nutrient_fraction_of_by_herb.zoopl._egested_foodcVSetDiat $([-])$, default $=0.25$

fDissMortZoo: 0.1 \# soluble_nutrient_fraction_of_died_zooplanktoncVSetGren ([-]), default $=0.1$

cSiDDiat: 0.15 \# Si/DW_ratio_of_daitomscVSetBlue (mgSi/mgDW), default $=0.15$ cNDDiatMin: $0.01 \quad$ \# minimum N/day ratio Diatoms $(\mathrm{mgN} / \mathrm{mgDW})$, default $=0.01$ cPDDiatMin: 0.0005 \# minimum P/day ratio Diatoms (mgP/mgDW), default $=0.0005$ cNDGrenMin: $0.02 \quad \#$ minimum N/day ratio greens $(\mathrm{mgN} / \mathrm{mgDW})$, default $=0.02$ cPDGrenMin: 0.0015 \# minimum P/day ratio greens (mgP/mgDW), default $=0.0015$ cNDBlueMin: 0.03 \# minimum N/day ratio Bluegreens (mgN/mgDW), default $=0.03$ cPDBlueMin: 0.0025 \# minimum P/day ratio Bluegreens $(\mathrm{mgP} / \mathrm{mgDW})$, default $=0.0025$ cNDBlueMax: 0.15 \# max. N/day ratio Bluegreens $(\mathrm{mgN} / \mathrm{mgDW})$, default $=0.15$ cNDDiatMax: $0.05 \quad \# \max . \mathrm{N} /$ day ratio Diatoms $(\mathrm{mgN} / \mathrm{mgDW})$, default $=0.05$ cNDGrenMax: 0.1 \# max. N/day goratio greens $(\mathrm{mgN} / \mathrm{mgDW})$, default $=0.1$ cPDBlueMax: 0.025 \# max. P/day ratio blue-greens (mgP/mgDW), default $=0.025$ cPDDiatMax: $0.005 \# \max . \mathrm{P} /$ day ratio Diatoms $(\mathrm{mgP} / \mathrm{mgDW})$, default $=0.005$ cPDGrenMax: 0.015 \# max. P/day ratio greens $(\mathrm{mgP} / \mathrm{mgDW})$, default $=0.01$ coupling:

diatom_as_food_DW: phytoplankton_water/sDDiatW green_as_food_DW: phytoplankton_water/sDGrenW blue_as_food_DW: phytoplankton_water/sDBlueW diatom_as_food_N: phytoplankton_water/sNDiatW green_as_food_N: phytoplankton_water/sNGrenW blue_as_food_N: phytoplankton_water/sNBlueW diatom_as_food_P: phytoplankton_water/sPDiatW green_as_food_P: phytoplankton_water/sPGrenW blue_as_food_P: phytoplankton_water/sPBlueW detritus_DW_pool_water: abiotic_water/sDDetW

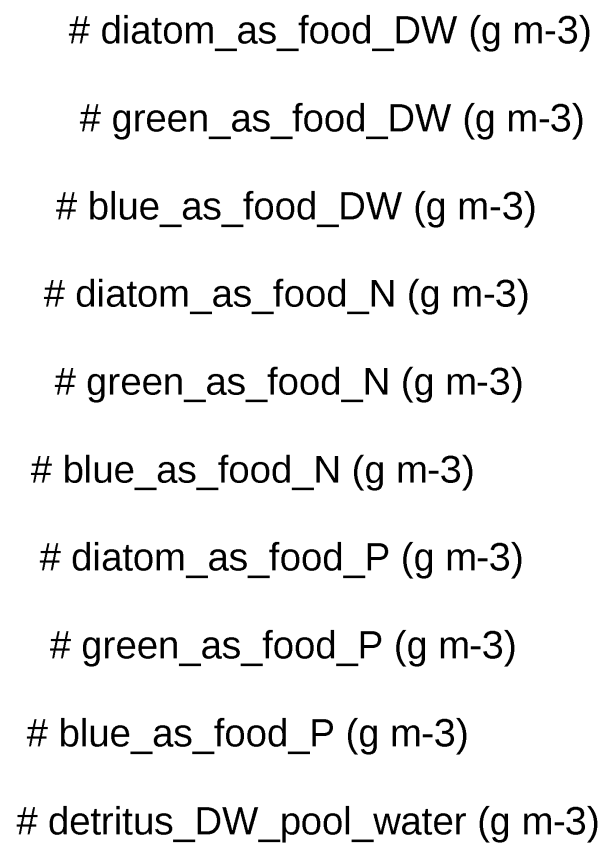


detritus_N_pool_water: abiotic_water/sNDetW

detritus_P_pool_water: abiotic_water/sPDetW

detritus_Si_pool_water: abiotic_water/sSiDetW

NH4_pool_water: abiotic_water/sNH4W

NO3_pool_water: abiotic_water/sNO3W

PO4_pool_water: abiotic_water/sPO4W
\# detritus_N_pool_water (g m-3)

\# detritus_P_pool_water (g m-3)

\# detritus_Si_pool_water (g m-3)

\# NH4_pool_water (g m-3)

\# NO3_pool_water (g m-3)

\# PO4_pool_water (g m-3)

\section{S3.7 FABM-PCLake_fish}

model: fish

long_name: fish

initialization:

sDFiJv: 0.25 \# juvenile fish DW in water ( $\mathrm{g} \mathrm{m}-3$ )

sPFiJv: 0.0055 \# juvenile fish $\mathrm{P}$ in water (g m-3)

sNFiJv: 0.025 \# juvenile fish $\mathrm{N}$ in water $(\mathrm{g} \mathrm{m}-3)$

sDFiAd: $1.0 \quad$ \# adult fish DW in water ( $\mathrm{g} \mathrm{m}-3)$

sPFiAd: 0.022 \# adult fish $\mathrm{P}$ in water (g m-3)

sNFiAd: $0.1 \quad$ \# adult fish $\mathrm{N}$ in water (g m-3)

sDPisc: 0.005 \# piscivorous fish DW in water ( $\mathrm{g} \mathrm{m}-3)$

parameters:

kMigrFish: 0.001 \# fish migration rate $(\mathrm{d}-1)$, default $=0.001$

cDFiJvIn: $0.005 \quad$ \# external fish density (gDW m-2), default $=0.005$

cDFiAdln: 0.005 \# external fish density (gDW m-2), default $=0.005$

cDPiscln: $0.001 \quad$ \# external Pi_sc. density (gDW m-2), default $=0.001$

kMigrPisc: 0.001 \# Pi_sc. migration rate $(\mathrm{d}-1)$, default $=0.001$

fDBone: $0.35 \quad$ \# fraction of fish $\mathrm{C}$ fixed in bones and scales $([-])$, default $=0.35$

fPBone: $0.5 \quad$ \# fraction of fish $P$ fixed in bones and scales $([-])$, default $=0.5$

cDCarrFish: 15.0 \# carrying capacity of fish (gDW m-2), default $=15.0$

fDissEgesFish: 0.25 \# soluble nutrient fraction of by fish egested food $([-])$, default $=0.25$ 
fDissMortFish: 0.1 \# soluble nutrient fraction of died fish(excl. bones and scales ([-]), default $=0.1$

cTmOptFish: $25.0 \quad$ \# optimum temp. of fish $(\diamond \mathrm{C})$, default $=25.0$

cSigTmFish: $10.0 \quad$ \# temperature constant of fish(sigma in Gaussian curve) $(\diamond \mathrm{C})$, default $=10.0$

cDayReprFish: 120.0 \# reproduction date of fish ([-]), default $=120.0$

fReprFish: $0.02 \quad$ \# yearly reproduction fraction of adult fish, daily rate ([-]), default = 0.02

fAgeFish: $0.5 \quad$ \# yearly ageing fraction of young fish, .daily rate $([-])$, default $=0.5$ kDAssFiJv: 0.12 \# maximum assimilation rate of young fish (d-1), default $=0.12$ hDZooFiJv: 1.25 \# half-saturating zooplankton biomass for young fish predation ( $\mathrm{g} \mathrm{m}-2)$, default $=1.25$

fDAssFiJv: $0.4 \quad$ \# C assimilation efficiency of young fish ([-]), default $=0.4$ kDRespFiJv: 0.01 \# maintenance respiration constant of young fish $(\mathrm{d}-1)$, default $=0.01$ kMortFiJv: 0.00137 \# specific mortality of young fish (d-1), default $=0.00137$ kDRespFiAd: $0.004 \quad \#$ maintenance respiration constant of adult fish (d-1), default $=0.004$ kMortFiAd: $0.00027 \quad$ \# specific mortality of adult fish (d-1), default $=0.00027$ cDCarrPiscMax: 1.2 \# maximum carrying capacity of Pi_sc (gDW m-2), default $=1.2$ cDCarrPiscMin: 0.1 \# minimum carrying capacity of Pi_sc (gDW m-2), default $=0.1$ cDCarrPiscBare: 0.1 \# carrying capacity of Pi sc for lake without marsh zone (gDW m-2), default $=0.1$ cDPhraMinPisc: 50.0 \# min. reed biomass for Pi_sc (gDW m-2), default $=50.0$ cCovVegMin: $40.0 \quad$ \# min. subm.veg. coverage for Pi_sc (\%), default $=40.0$ cRelPhraPisc: 0.075 \# rel. Pi_sc density per reed if subm.veg. absent (gDW m-2), default $=0.075$

cRelVegPisc: 0.03 \# extra rel. Pi_sc density perreed if aCovVeg > cCovVegMin (gDW $\mathrm{m}-2)$, default $=0.03$ 
kDAssPisc: $0.025 \quad$ \# maximum assimilation rate $(\mathrm{d}-1)$, default $=0.025$

hDVegPisc: $5.0 \quad$ \# half-sat. vegetation biomass for Pi_sc growth $(\mathrm{g} \mathrm{m}-2)$, default $=5.0$

hDFishPisc: $1.0 \quad$ \# half-saturating DFish for Pi_sc predation $(\mathrm{g} \mathrm{m}-2)$, default $=1.0$

fDAssPisc: $0.4 \quad$ \# C ass. efficiency of Pi_sc ([-]), default $=0.4$

fDissEgesPisc: $0.25 \quad$ \# soluble $P$ fraction of by fish egested food $([-])$, default $=0.25$

kDRespPisc: $0.005 \quad \#$ maint. respiration constant of Pi_sc (d-1), default $=0.005$

kMortPisc: 0.00027 \# specific mortality of Pi_sc (d-1), default $=0.00027$

fDissMortPisc: 0.1 \# soluble nutrient fraction of died Pi_sc(excl. bones and scales ([-]), default $=0.1$

cTmOptPisc: $25.0 \quad$ \# optimum temp. of Pi_sc $(\diamond \mathrm{C})$, default $=25.0$

cSigTmPisc: 10.0 \# temperature constant of Pi_sc(sigma in Gaussian curve) $(\diamond \mathrm{C})$,

default $=10.0$

cPDFishRef: $0.022 \quad$ \# reference P/C ratio of Fish $(\mathrm{mgP} / \mathrm{mgDW})$, default $=0.022$

cNDFishRef: $0.1 \quad$ \# reference $\mathrm{N} / \mathrm{C}$ ratio of Fish $(\mathrm{mgN} / \mathrm{mgDW})$, default $=0.1$

cPDPisc: $0.022 \quad$ \# reference P/C ratio of Pi_sc $(\mathrm{mgP} / \mathrm{mgDW})$, default $=0.022$

cNDPisc: $0.1 \quad$ \# reference N/C ratio of Pi_sc $(\mathrm{mgN} / \mathrm{mgDW})$, default $=0.1$

\# Fish manipulation switches

Manipulate_FiAd : false. \# Turned on/off external fish manipulation rate, + values,remove the fish;- values, add fish

Manipulate_FiJv : false. \# Turned on/off external fish manipulation rate, + values,remove the fish;- values, add fish

Manipulate_Pisc : false. \# Turned on/off external fish manipulation rate, + values, remove the fish;- values, add fish

\section{coupling:}

detritus_DW_pool_water: abiotic_water/sDDetW

detritus_N_pool_water: abiotic_water/sNDetW

detritus_P_pool_water: abiotic_water/sPDetW
\# detritus_DW_pool_water (g m-3)

\# detritus_N_pool_water (g m-3)

\# detritus_P_pool_water (g m-3) 
NH4_pool_water: abiotic_water/sNH4W

PO4_pool_water: abiotic_water/sPO4W

env_correction_adfish: zoobenthos/tDEnvFiAd

submerged_vegetation: macrophytes/aDSubVeg

food_limit_function_adfish: zoobenthos/aDSatFiAd \# food_limit_function_adfish ([-])

Zooplankton_D_Food: zooplankton/sDZoo

Zooplankton_N_Food: zooplankton/sNZoo

Zooplankton_P_Food: zooplankton/sPZoo

\section{S3.8 FABM-PCLake_zoobenthos}

model: pclake_zoobenthos

long_name: zoobenthos

initialization:

sDBent: 1.0 \# zoobenthos_DW_in_lake_sediment

sPBent: 0.01 \# zoobenthos_P_in_lake_sediment

sNBent: 0.07 \# zoobenthos_N_in_lake_sediment

parameters:

cDBentIn: $0.01 \quad \#$ gDW/m2,external_zoobenthos_density

cDCarrBent: $10.0 \quad$ \# gDW/m2,carrying_capacity_of_zoobenthos

cDCarrFish: $15 \quad \#$ gDW/m2,carrying_capacity_of_fish(:_100_gFW/m2,Grimm_1983)

cNDBentRef: $0.07 \quad \# \mathrm{mgN} / \mathrm{mgDW}$, reference_N/C_ratio_of_zoobenthos

cNDFishRef: $0.1 \quad \# \mathrm{mgN} / \mathrm{mgDW}$,reference_N/C_ratio_of_Fish

cPDBentRef: $0.01 \quad \#$ mgP/mgDW,reference_P/C_ratio_of_zoobenthos

cPDFishRef: $0.022 \quad$ \# mgP/mgDW,reference_P/C_ratio_of_Fish

cRelVegFish: $0.009 \quad \#-$,decrease_of_fish_feeding_per_\%_vegetation_cover(max._0.01)

cSiDDiat: 0.15 \# mgSi/mgDW, Si/DW_ratio_of_daitoms

cSigTmBent: $16.0 \quad$ \#

${ }^{\circ} \mathrm{C}$,temperature_constant_of_zoobenthos(sigma_in_Gaussian_curve)

cSigTmFish: $10 \quad \#{ }^{\circ} \mathrm{C}$,temperature_constant_of_fish(sigma_in_Gaussian_curve) 
cTmOptBent: $25.0 \quad \# \quad{ }^{\circ} \mathrm{C}$, optimum_temp._of_zoobenthos

cTmOptFish: $25 \quad \#{ }^{\circ} \mathrm{C}$, optimum_temp._of_fish

fDAssBent: $0.3 \quad$ \# -,C_ass._efficiency_of_zoobenthos

fDAssFiAd: $0.4 \quad$ \# -,C_assimilation_efficiency_of_adult_fish

fDissEgesBent: 0.25 \# -,soluble_nutrient_fraction_of_by_zoobenthos_egested_food

fDissEgesFish: 0.25 \# -,soluble_nutrient_fraction_of_by_fish_egested_food

fDissMortBent: $0.1 \quad \# \quad$-,soluble_P_fraction_of_died_zoobenthos_P

hDBentFiAd: $2.5 \quad \#$ g/m2,half-

saturating_zoobenthos_biomass_for_adult_fish_predation

hDFoodBent: $200.0 \quad$ \# g/m2,half-saturating_food_for_zoobenthos

kDAssBent: 0.1 \# day-1, maximum_assimilation_rate

kDAssFiAd: 0.06 \# day-1,maximum_assimilation_rate_of_adult_fish

kDRespBent: 0.005 \# day-1,maint._respiration_constant_of_zoobenthos

kDRespFiAd: 0.004 \# day-1,maintenance_respiration_constant_of_adult_fish

kMigrBent: $0.001 \quad \#$ day-1,zoobenthos_migration_rate

kMortBent: $0.005 \quad$ \# day-1,mortality_constant_of_zoobenthos

kMortFiAd: 0.00027 \# day-1,specific_mortality_of_adult_fish(:_0.1_y-1)

cNDBlueMax: $0.15 \quad \#$ mgN/mgDW, max. N/day ratio bluegreens

cNDBlueMin: $0.03 \quad \# \mathrm{mgN} / \mathrm{mgDW}$, minimum N/day ratio bluegreens

cNDDiatMax: $0.05 \quad \# \mathrm{mgN} / \mathrm{mgDW}$, max. N/day ratio diatoms

cNDDiatMin: $0.01 \quad \# \mathrm{mgN} / \mathrm{mgDW}$, minimum N/day ratio diatoms

cNDGrenMax: $0.1 \quad \#$ mgN/mgDW, max. N/day ratio greens

cNDGrenMin: $0.02 \quad \# \mathrm{mgN} / \mathrm{mgDW}$, minimum N/day ratio greens

cPDBlueMax: 0.025 \# mgP/mgDW, max. P/day ratio bluegreens

cPDBlueMin: 0.0025 \# mgP/mgDW, minimum P/day ratio bluegreens

cPDDiatMax: $0.05 \quad \# \mathrm{mgP} / \mathrm{mgDW}$, max. P/day ratio diatoms

cPDDiatMin: 0.005 \# mgP/mgDW, minimum P/day ratio diatoms

cPDGrenMax: 0.015 \# mgP/mgDW, max. P/day ratio greens 
cPDGrenMin: 0.0015 \# mgP/mgDW, minimum P/day ratio greens coupling:

diatom_as_food_DW: phytoplankton_sediment/sDDiatS green_as_food_DW: phytoplankton_sediment/sDGrenS blue_as_food_DW: phytoplankton_sediment/sDBlueS diatom_as_food_N: phytoplankton_sediment/sNDiatS green_as_food_N: phytoplankton_sediment/sNGrenS blue_as_food_N: phytoplankton_sediment/sNBlueS diatom_as_food_P: phytoplankton_sediment/sPDiatS green_as_food_P: phytoplankton_sediment/sPGrenS blue_as_food_P: phytoplankton_sediment/sPBlueS detritus_DW_pool_sediment: abiotic_sediment/sDDetS detritus_P_pool_sediment: abiotic_sediment/sPDetS detritus_N_pool_sediment: abiotic_sediment/sNDetS detritus_Si_pool_sediment: abiotic_sediment/sSiDetS NH4_pool_sediment: abiotic_sediment/sNH4S NO3_pool_sediment: abiotic_sediment/sNO3S PO4_pool_sediment: abiotic_sediment/sPO4S adult_fish_biomass:fish/sDFiAd adult_fish_nitrogen: fish/sNFiAd adult_fish_phosphorus: fish/sPFiAd NH4_pool_water: abiotic_water/sNH4W PO4_pool_water: abiotic_water/sPO4W DDet_pool_water: abiotic_water/sDDetW NDet_pool_water: abiotic_water/sNDetW PDet_pool_water: abiotic_water/sPDetW vegetation_coverage: macrophytes/aCovVeg young_fish_biomass: fish/sDFiJv 


\section{S3.9FABM-PCLake_auxiliary}

model: pclake_auxiliary

long_name: extra_processes

parameters:

cDepthS: $0.1 \quad$ \# sediment depth [m]

cThetaSet: $1.01 \quad \# 1 / \mathrm{e}^{\wedge 0} \mathrm{C}$, temp. parameter of sedimentation

kVegResus: $0.01 \quad$ \# m2/gDW, rel. resuspension reduction per g vegetation

kTurbFish: $1.0 \quad$ \# g/g fish/day, relative resuspension by adult fish browsing

cSuspRef: $0.5 \quad$ \# reference suspended matter function [-]

cSuspMin: $6.1 \quad$ \# minimum value of logistic function

cSuspMax: $25.2 \quad$ \# maximum value of logistic function

cSuspSlope: 2.1 \# slope of logistic function

hDepthSusp: $2.0 \quad$ \# half-sat. value of depth in logistic function

cFetchRef: 1000.0 \# reference fetch [m]

cFetch: $1000.0 \quad$ \# fetch [m],the length of the lake in the prevailing wind direction

fLutum: $0.1 \quad$ \# lutum content of inorg. matter

fLutumRef: 0.2 \# reference lutum fraction

bPorS: $0.847947 \quad$ \# porosity [m3 water m-3 sediment]

kResusPhytMax: 0.25 \# day-1,max._phytopl._resuspension

cResusPhytExp: -0.379 \# (gDW/m2/day)-1,exp._par._for_phytopl._resuspension

cVSetIM: $1.0 \quad$ \# m/day, max. sedimentation velocity of inert org. matter

cVSetDet: $0.25 \quad$ \# m/day, max. sedimentation velocity of detritus

cVSetDiat: $0.5 \quad$ \# m/day, sedimentation velocity diatoms

cVSetGren: $0.2 \quad \#$ m/day, sedimentation velocity of greens

cVSetBlue: $0.06 \quad \# \mathrm{~m} /$ day, sedimentation velocity blue-greens

cRholM: $2500000.0 \quad$ \# g/m3 solid, density of sediment IM 
cRhoOM: $1400000.0 \quad \# \mathrm{~g} / \mathrm{m3}$, density of sediment detritus

fDOrgSoil: $0.1 \quad$ \# fraction soil organic matter

cPO4Ground: $0.1 \quad$ \# mgP/l, PO4 cone in groundwater

cNH4Ground: $1.0 \quad \# \mathrm{mgN} / \mathrm{l}, \mathrm{NH} 4$ cone in groundwater

cNO3Ground: $0.1 \quad \# \mathrm{mgN} / \mathrm{l}, \mathrm{NO} 3$ cone in groundwater

cTmOptFish: $25 \quad$ \# ${ }^{\circ} \mathrm{C}$,optimum_temp._of_fish

cSigTmFish: $10 \quad \#{ }^{\circ} \mathrm{C}$,temperature_constant_of_fish(sigma_in_Gaussian_curve)

CLoadPO4: $0.005 \quad$ \# gP/m2/day, constant, different from cLoadIM

cLoadNO3: $0.05 \quad$ \# gN/m2/day, constant

uQIn: $20.0 \quad$ \# mm/day, net water load rate, constant

cDErosTot: $0.1 \quad$ \# g/m2/day,Erosion_input_(tentative)

fSedErosIM: 0.95 \# instantly_sedimentating_fraction_of_IM

cNDBlueMax: $0.15 \quad \# \mathrm{mgN} / \mathrm{mgDW}$, max. N/day ratio bluegreens

cNDBlueMin: $0.03 \quad \# \mathrm{mgN} / \mathrm{mgDW}$, minimum N/day ratio bluegreens

cNDDiatMax: $0.05 \quad \# \mathrm{mgN} / \mathrm{mgDW}$, max. N/day ratio diatoms

cNDDiatMin: $0.01 \quad \# \mathrm{mgN} / \mathrm{mgDW}$, minimum N/day ratio diatoms

cNDGrenMax: $0.1 \quad \# \mathrm{mgN} / \mathrm{mgDW}$, max. N/day ratio greens

cNDGrenMin: $0.02 \quad \# \mathrm{mgN} / \mathrm{mgDW}$, minimum N/day ratio greens

cPDBlueMax: 0.025 \# mgP/mgDW, max. P/day ratio bluegreens

cPDBlueMin: $0.0025 \quad \# \mathrm{mgP} / \mathrm{mgDW}$, minimum P/day ratio bluegreens

cPDDiatMax: $0.005 \quad \# \mathrm{mgP} / \mathrm{mgDW}$, max. P/day ratio diatoms

cPDDiatMin: 0.0005 \# mgP/mgDW, minimum P/day ratio diatoms

cPDGrenMax: 0.015 \# mgP/mgDW, max. P/day ratio greens

cPDGrenMin: 0.0015 \# mgP/mgDW, minimum P/day ratio greens

coupling:

Ammonia_pool_in_water: abiotic_water/sNH4W

Nitrate_pool_in_water: abiotic_water/sNO3W

Phosphate_pool_in_water: abiotic_water/sPO4W 
Adsorbed_phosphorus_in_water: abiotic_water/sPAIMW

Oxygen_pool_in_water: abiotic_water/sO2W

Inorg_pool_in_water: abiotic_water/sDIMW

Detritus_DW_in_water: abiotic_water/sDDetW

Detritus_N_in_water: abiotic_water/sNDetW

Detritus_P_in_water: abiotic_water/sPDetW

Detritus_Si_in_water: abiotic_water/sSiDetW

Diatom_DW_in_water: phytoplankton_water/sDDiatW

Green_DW_in_water: phytoplankton_water/sDGrenW

Blue_DW_in_water: phytoplankton_water/sDBlueW

Diatom_N_in_water: phytoplankton_water/sNDiatW

Green_N_in_water: phytoplankton_water/sNGrenW

Blue_N_in_water: phytoplankton_water/sNBlueW

Diatom_P_in_water: phytoplankton_water/sPDiatW

Green_P_in_water: phytoplankton_water/sPGrenW

Blue_P_in_water: phytoplankton_water/sPBlueW

Ammonia_pool_in_sediment: abiotic_sediment/sNH4S

Nitrate_pool_in_sediment: abiotic_sediment/sNO3S

Phosphate_pool_in_sediment: abiotic_sediment/sPO4S

Adsorbed_phosphorus_in_sediment: abiotic_sediment/sPAIMS

Inorg_pool_in_sediment: abiotic_sediment/sDIMS

Detritus_DW_in_sediment: abiotic_sediment/sDDetS

Detritus_N_in_sediment: abiotic_sediment/sNDetS

Detritus_P_in_sediment: abiotic_sediment/sPDetS

Detritus_Si_in_sediment: abiotic_sediment/sSiDetS

Diatom_DW_in_sediment: phytoplankton_sediment/sDDiatS

Green_DW_in_sediment: phytoplankton_sediment/sDGrenS

Blue_DW_in_sediment: phytoplankton_sediment/sDBlueS 
Diatom_N_in_sediment: phytoplankton_sediment/sNDiatS

Green_N_in_sediment: phytoplankton_sediment/sNGrenS

Blue_N_in_sediment: phytoplankton_sediment/sNBlueS

Diatom_P_in_sediment: phytoplankton_sediment/sPDiatS

Green_P_in_sediment: phytoplankton_sediment/sPGrenS

Blue_P_in_sediment: phytoplankton_sediment/sPBlueS

vegetation_DW: macrophytes/sDVeg

adult_fish_DW: fish/sDFiAd

Detritus_abiotic_update: abiotic_sediment/tDAbioDetS

Detritus_from_algae: phytoplankton_sediment/tDPrimDetS

Detritus_from_vegetation: macrophytes/tDBedDetS

Detritus_from_zoobenthos: zoobenthos/tDWebDetS

Humus_DW_in_sediment: abiotic_sediment/sDHumS

Humus_N_in_sediment: abiotic_sediment/sNHumS

Humus_P_in_sediment: abiotic_sediment/sPHumS

Humus_abiotic_update: abiotic_sediment/tDAbioHumS

Zooplankton_DW: zooplankton/sDZoo

Zooplankton_P: zooplankton/sPZoo

Zooplankton_N: zooplankton/sNZoo

SiO2_pool_water: abiotic_water/sSiO2W

\section{S4. References}

Janse, J. H. 2005. Model studies on the eutrophication of shallow lakes and ditches. Jan $\mathrm{H}$. Janse, Ph.D. Thesis, Wageningen University, ISBN 90-8504-214-3, 378p. 